\title{
On the Uniqueness of Solutions of Stochastic Differential Equations with Singular Drifts
}

\author{
By \\ Satoshi TAKANOBU*
}

\section{§0. Introduction and Results}

Let $d \geqq 2$. Let $a, \tau: \mathbb{R}^{d} \rightarrow \mathbb{R}^{d} \otimes \mathbb{R}^{d}$ and $b, \beta: \mathbb{R}^{d} \rightarrow \mathbb{R}^{d}$ be bounded continuous functions and let $\mu(d \eta)$ be a nonnegative bounded measure on $\mathbb{R}^{1}$. We consider the following stochastic differential equation (SDE) with a singular drift:

$$
\begin{aligned}
d X^{i}(t)= & \sum_{j=1}^{d} a_{j}^{i}(X(t)) d B^{j}(t)+b^{i}(X(t)) d t \\
& +\sum_{j=1}^{d} \tau_{j}^{i}(X(t)) d M^{j}(t)+\beta^{i}(X(t)) d L_{t}^{\mu}\left(X^{d}\right) \quad i=1, \ldots, d,
\end{aligned}
$$

the precise formulation of which is given below. We are concerned with the existence and uniqueness of solutions of the SDE (0.1). This SDE was studied by Y. Ôshima [4], S. V. Anulova [1] and S. Takanobu [6]. Ôshima obtained the existence and uniqueness results in the case of Lipschitz continuous coefficients and general $\mu$ under the assumption that $\beta^{d} \equiv 0$. Anulova discussed the case $\mu(d \eta)=\delta_{0}(d \eta)$ but with general $\beta^{d}$ by the method of the submartingale problem and obtained the existence of solutions. Takanobu obtained the existence of solutions in a more general case of $\beta^{d}$ and $\mu$. Also it should be remarked that in one dimension Le Gall [3] considered an SDE like (0.1) and obtained the pathwise uniqueness of solutions.

The purpose of present paper is to obtain the uniqueness of solutions of (0.1) in the case of general $\beta^{d}$ but $\mu$ is restricted to a certain class of discrete measures: The case $\mu=\delta_{0}$ is the most typical case and actually, we consider only cases which can be reduced to this special case. In Ôshima's case, the method

Communicated by S. Matsuura, March 14, 1986.

* Department of Mathematics, Faculty of Science, Tokyo Metropolitan University, Setagaya, Tokyo 158, Japan. 
of skew product is a fundamental tool but, in our case, this method is not applicable and we have to exploit another method. For this, we use the method of decomposing the solution into pieces called excursions in $\mathbb{R}^{d} \backslash\left\{x ; x^{d}=0\right\}$. By showing that the point process formed of these excursions is uniquely represented by means of a Brownian motion and Poisson point process of Brownian excursions, we can clarify the structure of solutions and consequently, we can show the uniqueness of solutions. We remark that excursion point processes have been discussed in Watanabe [8] and [9] with somewhat different purposes.

Let $a, b, \tau, \beta$ and $\mu$ be as above. In the following, we enumerate several conditions considered in this paper on $a, b, \tau, \beta$ and $\mu$ :

$$
\begin{gathered}
\inf _{x \in \mathbb{R}^{d}} \sum_{j=1}^{d} a_{j}^{d}(x)^{2}>0 \\
a_{j}^{d}=\delta_{j}^{d} \quad j=1, \ldots, d, \quad b^{d}=0 \\
\tau_{j}^{d}=0 \quad j=1, \ldots, d \\
\sup _{\xi \in \mathbb{R}^{d-1}}\left|\beta^{d}(\xi, \eta) \mu\{\eta\}\right| \leqq 1 \quad \eta \in \mathbb{R}^{1} \\
\mu(d \eta)=\sum_{k \in \mathbb{Z}} c_{k} \delta_{a_{k}}(d \eta)
\end{gathered}
$$

where $c_{k} \geqq 0(k \in \mathbb{Z}), \sum_{k \in Z} c_{k}<+\infty, \inf _{k \in Z}\left(a_{k+1}-a_{k}\right)>0$ and $\sup _{k \in Z}\left(a_{k+1}-a_{k}\right)$ $<+\infty$

$$
\begin{aligned}
& \mu(d \eta)=\delta_{0}(d \eta) \\
& a, b, \tau \text { and } \beta \text { are Lipschitz continuous. }
\end{aligned}
$$

First of all we give the formulation of SDE (0.1). By a solution of (0.1) we mean a system of continuous stochastic processes $\mathfrak{X}=\left[X(t)=\left(X^{1}(t), \ldots, X^{d}(t)\right)\right.$, $\left.B(t)=\left(B^{1}(t), \ldots, B^{d}(t)\right), M(t)=\left(M^{1}(t), \ldots, M^{d}(t)\right)\right]$ defined on a filtered probability space $\left(\Omega, \mathscr{F}, P, \mathscr{F}_{t}\right)$ such that

(i) $X(t)$ is a system of $\mathscr{F}_{t}$-semimartingales,

(ii) $[B(t), M(t)]$ is a system of $\mathscr{F}_{t}$-martingales with $B(0)=M(0)=0$ such that $\left\langle B^{i}, B^{j}\right\rangle(t)=\delta_{j}^{i} t,\left\langle B^{i}, M^{j}\right\rangle(t)=0$ and $\left\langle M^{i}, M^{j}\right\rangle(t)=\delta_{j}^{i} L_{t}^{\mu}\left(X^{d}\right)$,

(iii) with probability one

$$
\begin{aligned}
& X^{i}(t)=X^{i}(0)+\sum_{j=1}^{d} \int_{0}^{t} a_{j}^{i}(X(s)) d B^{j}(s)+\int_{0}^{t} b^{i}(X(s)) d s \\
& +\sum_{j=1}^{d} \int_{0}^{t} \tau_{j}^{i}(X(s)) d M^{j}(s)+\int_{0}^{t} \beta^{i}(X(s)) d L_{s}^{\mu}\left(X^{d}\right) \quad i=1, \ldots, d
\end{aligned}
$$


where $\left[L_{t}^{\eta}\left(X^{d}\right) ; t \geqq 0, \eta \in \mathbb{R}^{1}\right]$ denotes the local time of a continuous $\mathscr{F}_{t^{-}}$ semimartingale $X^{d}(t)$ and $L_{t}^{\mu}\left(X^{d}\right)=\int_{-\infty}^{+\infty} \mathbb{L}_{t}^{\eta}\left(X^{d}\right) \mu(d \eta)$.

For a probability measure $\lambda(d x)$ on $\mathbb{R}^{d}$, we denote by $\mathbb{S}_{\lambda}[a, b, \tau, \beta, \mu]$ the totality of all solutions $\mathfrak{X}=[X(t), B(t), M(t)]$ of $(0.1)$ for $a, b, \tau, \beta$ and $\mu$ such that the probability law of $X(0)$ coincides with $\lambda(d x)$. We say that the uniqueness holds for $\mathbb{S}_{\lambda}[a, b, \tau, \beta, \mu]$ if whenever $\mathfrak{x}$ and $\mathfrak{X}^{\prime}$ are any two elements of $\mathbb{S}_{\lambda}[a, b, \tau, \beta, \mu]$, then the probability laws of $\mathbb{X}(\cdot)$ and $X^{\prime}(\cdot)$ coincide.

The results in this paper are summarized in the following.

Theorem $\mathbb{A}$ (Existence theorem). Suppose that $a, b, \tau, \beta$ and $\mu$ satisfy $(0.2),(0.3),(0.4)$ and $(0.5)$. Then for any probability measure $\lambda(d x)$ on $\mathbb{R}^{d}$, $\mathbb{S}_{\lambda}[a, b, \tau, \beta, \mu]$ is nonempty.

Theorem $\mathbb{B}$ (Uniqueness theorem). Suppose that $a, b, \tau, \beta$ and $\mu$ satisfy $(0.2),(0.3),(0.4),(0.5)$ and (0.6). Then for any probability measure $\lambda(d x)$ on $\mathbb{R}^{d}, \mathbb{S}_{\lambda}[a, b, \tau, \beta, \mu]$ is nonempty and its uniqueness holds.

Remark 0.1. In [6], Takanobu proved the existence theorem by assuming $(0.2),(0.3)$ and

$$
\sup _{\xi \in \mathbb{R}^{d-1}}\left|\beta^{d}(\xi, \eta) \mu\{\eta\}\right|<1 \quad \eta \in \mathbb{R}^{1} .
$$

Also, Anulova's assumptions are $(0.2),(0.3)$ and $(0.4)$ with $\mu(d \eta)=\delta_{0}(d \eta)$. Thus Theorem $\mathrm{A}$ is an extension of Anulova's, and, under the condition (0.5), is also an extension of Takanobu's. In [4], Ôshima assumed that $(0.2),(0.3)$, $(0.6)$ and $\beta^{d} \equiv 0$.

In $\S 2$ we shall prove Theorem $B$ in the case of $(0.5)^{\prime}$ and the general case will be proven by reducing it to the case $(0.5)^{\prime}$ in $\S 4 . \quad \S 1$ is devoted to preparing some propositions which play an important role in $\S 2$. In $\S 3$ we shall prove Theorem A.

\section{§1. Prellimingmi̊es}

Let $a: \mathbb{R}^{d} \rightarrow \mathbb{R}^{d} \otimes \mathbb{R}^{d}$ and $b: \mathbb{R}^{d} \rightarrow \mathbb{R}^{d}$ be bounded Lipschitz continuous functions such that $a_{j}^{d}=\delta_{j}^{d}(j=1, \ldots, d)$ and $b^{d}=0$. We denote the $\mathbb{L i p s c h i t z}$ constants of $a$ and $b$ by $K_{a}$ and $K_{b}$, respectively. Let $\mathscr{W}^{d}$ be the set of all continuous paths $w:[0, \infty) \rightarrow \mathbb{R}^{d}$.

$\S \S 1.1$. For each $x \in \mathbb{R}^{d}$, let $\mathbb{P}^{x}$ be the unique solution to the martingale 
problem for $\mathscr{L}$ starting from $x$, where

$$
\mathscr{L}=\frac{1}{2} \sum_{i, j=1}^{d}\left(a a^{*}\right)^{i j}(x) \frac{\partial^{2}}{\partial x^{i} \partial x^{j}}+\sum_{i=1}^{d} b^{i}(x) \frac{\partial}{\partial x^{i}} .
$$

Setting $\sigma_{0}(w)=\inf \left\{t \geqq 0 ; w^{d}(t)=0\right\}$ for $w \in \mathscr{W}^{d}$, we define a probability measure $\stackrel{\circ}{P}^{x}$ on $\mathscr{W}^{d}$ by

$$
\stackrel{\circ}{P}^{x}(*)=P^{x}\left\{w ; w\left(\cdot \wedge \sigma_{0}(w)\right) \in *\right\} .
$$

$\S \S 1.2$. We introduce the following subspaces of $\mathscr{W}^{d}$ :

$\mathscr{W}_{\partial}^{d, \pm}=$ the family of all $w \in \mathscr{W}^{d}$ such that (i) $w^{d}(0)=0$ and (ii) there exists a positive $\sigma(w)$ such that $w^{d}(t) \in{\stackrel{\circ}{\boldsymbol{R}_{ \pm}}}_{ \pm}$for $0<t<\sigma(\omega)$ and $w(t)=w(\sigma(w))$ $\in \mathbb{R}^{d-1} \times\{0\}$ for $t \geqq \sigma(w)$,

$\mathscr{W}_{0}^{d, \pm}=$ the subfamily of $\mathscr{W}_{\partial}^{d, \pm}$ such that $w(0)=0$,

$$
\begin{aligned}
& \mathscr{W}_{\partial}^{d}=\mathscr{W}_{\partial}^{d,+} \cup \mathscr{W}_{\partial}^{d,-}, \\
& \mathscr{W}_{0}^{d}=\mathscr{W}_{0}^{d,+} \cup \mathscr{W}_{0}^{d,-}
\end{aligned}
$$

where $\mathbb{R}_{+}$and $\mathbb{R}_{-}$denote $[0, \infty)$ and $(-\infty, 0]$, respectively. Let $\mathscr{B}\left(\mathscr{W}_{0}^{d, \pm}\right)$, $\mathscr{B}\left(\mathscr{W}_{\partial}^{d, \pm}\right), \mathscr{B}\left(\mathscr{W}_{0}^{d}\right), \ldots$ be the $\sigma$-fields on $\mathscr{W}_{0}^{d, \pm}, \mathscr{W}_{\partial}^{d, \pm}, \mathscr{W}_{0}^{d}, \ldots$ generated by Borel cylinder sets.

$\S \S 1.2 .1$. Let

$$
\begin{aligned}
K^{d, \pm}(t, x) & =\left(\prod_{i=1}^{d-1} \frac{1}{\sqrt{2 \pi t}} \exp \left\{-\left(x^{i}\right)^{2} / 2 t\right\}\right) \sqrt{\frac{2}{\pi t^{3}}}\left( \pm x^{d}\right) \exp \left\{-\left(x^{d}\right)^{2} / 2 t\right\} \\
\check{p}^{d}(t, x, y) & =\left(\prod_{i=1}^{d-1} \frac{1}{\sqrt{2 \pi t}} \exp \left\{-\left(x^{i}-y^{i}\right)^{2} / 2 t\right\}\right) \\
& \times \frac{1}{\sqrt{2 \pi t}}\left(\exp \left\{-\left(x^{d}-y^{d}\right)^{2} / 2 t\right\}-\exp \left\{-\left(x^{d}+y^{d}\right)^{2} / 2 t\right\}\right)
\end{aligned}
$$

for $t>0$ and $x, y \in \boldsymbol{R}^{d-1} \times \boldsymbol{R}_{ \pm}$. Then there exists a unique $\sigma$-finite measure $Q^{d, \pm}$ on $\left(\mathscr{W}_{0}^{d, \pm}, \mathscr{B}\left(\mathscr{W}_{a}^{d, \pm}\right)\right)$ such that

$$
\begin{aligned}
Q^{d, \pm}\left\{w ; w\left(t_{1}\right) \in E_{1}, \ldots, w\left(t_{m}\right) \in E_{m}, \sigma(w)>t_{m}\right\} \\
\quad=\int_{E_{1}} d x_{1} \int_{E_{2}} d x_{2} \cdots \int_{E_{m}} d x_{m} K^{d, \pm}\left(t_{1}, x_{1}\right) \prod_{i=1}^{m-1} \grave{p}^{d}\left(t_{i+1}-t_{i}, x_{i}, x_{i+1}\right)
\end{aligned}
$$

for $0<t_{1}<\cdots<t_{m}$ and $E_{i} \in \mathscr{B}\left(\mathbb{R}^{d-1} \times \boldsymbol{R}_{ \pm}\right)$(cf. [9] or p. 124-125 in [2]).

Since $\mathscr{B}\left(\mathscr{W}_{0}^{d}\right) \cap \mathscr{W}_{0}^{d, \pm}=\mathscr{B}\left(\mathscr{W}_{0}^{d, \pm}\right)$, we can define a $\sigma$-finite measure $Q^{d}$ on $\left(\mathscr{W}_{0}^{d}, \mathscr{B}\left(\mathscr{W}_{0}^{d}\right)\right)$ as follows :

$$
Q^{d}(*)=Q^{d,+}\left(* \cap \mathscr{W}_{0}^{d,+}\right)+Q^{d,-}\left(* \cap \mathscr{W}_{0}^{d,-}\right) \quad * \in \mathscr{B}\left(\mathscr{W}_{0}^{d}\right)
$$


Clearly $w^{i}(t) \in L^{2}\left(\mathscr{W}_{0}^{d}, Q^{d}\right)$ for $t>0$ and $i=1, \ldots, d$. Moreover, denoting by $\mathscr{B}_{t}\left(\mathscr{W}_{0}^{d}\right)$ the sub $\sigma$-field of $\mathscr{B}\left(\mathscr{W}_{0}^{d}\right)$ generated by Borel cylinder sets up to time $t$, we see easily that if $0<t<t^{\prime}$ and $H$ is $\mathscr{B}_{t}\left(\mathscr{W}_{0}^{d}\right)$-measurable and belongs to $L^{2}\left(\mathscr{W}_{0}^{d}\right.$, $\left.Q^{d}\right) \cap L^{\infty}\left(\mathscr{W}_{0}^{d}, Q^{d}\right)$, then

$$
\begin{aligned}
& \int_{\mathscr{Y}}^{d}\left(w^{i}\left(t^{\prime}\right)-w^{i}(t)\right) H(w) Q^{d}(d w)=0 \quad i=1, \ldots, d \\
& \int_{\mathscr{V}_{0}^{d}}\left(w^{i}\left(t^{\prime}\right)-w^{i}(t)\right)\left(w^{j}\left(t^{\prime}\right)-w^{j}(t)\right) H(w) Q^{d}(d w) \\
& \quad=\delta_{j}^{i} \int_{\mathscr{V}_{0}^{d}}\left\{t^{\prime} \wedge \sigma(w)-t \wedge \sigma(w)\right\} H(w) Q^{d}(d w) \quad i, j=1, \ldots, d .
\end{aligned}
$$

By virtue of this property of $Q^{d}$, for a $\mathscr{B}_{t}\left(\mathscr{W}_{0}^{d}\right)$-progressively measurable process $f(t, w)$ satisfying

$$
\int_{\mathscr{V}_{0}^{d}} Q^{d}(d w) \int_{0}^{t \wedge \sigma(w)} f(s, w)^{2} d s<+\infty \quad t \geqq 0,
$$

we can define the stochastic integral with respect to $Q^{d}$

$$
\mathbb{I}^{i}(f)(t)=\int_{0}^{t} f(s, w) d w^{i}(s) \quad i=1, \ldots, d
$$

in exactly the same way as ordinary Itô's integral (cf. [9]). Then this stochastic integral has the following properties:

(i) For almost all $w\left(Q^{d}\right), \quad t \mapsto \mathbb{I}^{i}(f)(t)$ is continuous, $\mathbb{I}^{i}(f)(0)=0$ and $\mathbb{Z}^{i}(f)(t)=\mathbb{H}^{i}(f)(t \wedge \sigma(w))$ for $t \geqq 0$.

(ii) $\mathbb{I}^{i}(f)(t) \in L^{2}\left(\mathscr{W}_{0}^{d}, Q^{d}\right)$ for $t \geqq 0$.

(iii) If $f^{i}(u, w)$ is a $\mathscr{B}_{u}\left(\mathscr{W}_{0}^{d}\right)$-progressively measurable process satisfying (1.2) $(i=1, \ldots, d), 0<t<t^{\prime}$ and $H$ is $\mathscr{B}_{t}\left(\mathscr{W}_{0}^{d}\right)$-measurable and an element of $L^{2}\left(\mathscr{W}_{0}^{d}, Q^{d}\right) \cap L^{\infty}\left(\mathscr{W}_{0}^{d}, Q^{d}\right)$, then

$$
\begin{aligned}
& E^{Q^{d}}\left[\left(\mathbb{I}^{i}\left(f^{i}\right)\left(t^{\prime}\right)-\mathbb{H}^{i}\left(f^{i}\right)(t)\right) H\right]=0 \quad i=1, \ldots, d \\
& E^{Q^{d}}\left[\left(\mathbb{I}^{i}\left(f^{i}\right)\left(t^{\prime}\right)-\mathbb{I}^{i}\left(f^{i}\right)(t)\right)\left(\mathbb{H}^{j}\left(f^{j}\right)\left(t^{\prime}\right)-\mathbb{I}^{j}\left(f^{j}\right)(t)\right) H\right] \\
& \quad=\delta_{j}^{i} E^{Q^{d}}\left[\int_{t \wedge \sigma}^{t^{\prime} \wedge \sigma} f^{i}(s) f^{j}(s) d s H\right] \quad i, j=1, \ldots, d
\end{aligned}
$$

where $E^{Q^{d}}$ stands for the integration by $Q^{d}$. We here note the fundamental inequality of Burkholder-Davis-Gundy type for the stochastic integral with respect to $Q^{d}$ : For $p>0$ and $T>0$

$$
\begin{gathered}
c_{p} E^{Q^{d}}\left[\max _{0 \leqq t \leqq T}\left|\int_{0}^{t} f(s, w) d w^{i}(s)\right|^{2 p}\right] \leqq E^{Q^{d}}\left[\left(\int_{0}^{T \wedge \sigma} f(s)^{2} d s\right)^{p}\right] \\
\leqq C_{p} E^{Q^{d}}\left[\max _{0 \leqq t \leqq T}\left|\int_{0}^{t} f(s, w) d w^{i}(s)\right|^{2 p}\right] \quad i=1, \ldots, d
\end{gathered}
$$


where $c_{p}$ and $C_{p}$ are positive universal constants depending only on $p$. This can be derived from the above property (iii).

$\S \S 1.2 .2$. For $\xi \in \mathbb{R}^{d-1}$ and $c \geqq 0$, we now consider the following $\mathrm{SDE}$ with respect to $Q^{d}$ :

$$
\begin{aligned}
\Xi^{i}(t, w)=\xi^{i} & +c \sum_{j=1}^{d} \int_{0}^{t} a_{j}^{i}\left(\Xi(s, w), c w^{d}(s)\right) d w^{j}(s) \\
& +c^{2} \int_{0}^{t \wedge \sigma(w)} b^{i}\left(\Xi(s, w), c w^{d}(s)\right) d s \quad i=1, \ldots, d-1
\end{aligned}
$$

where $\Xi(t, w)=\left(\Xi^{1}(t, w), \ldots, \Xi^{d-1}(t, w)\right)$ (cf. [9]). Since $a$ and $b$ are bounded Lipschitz continuous, this $\mathrm{SDE}$ is uniquely solvable, and hence we denote the unique solution by $\Xi_{c, \xi}$. Clearly $\Xi_{c, \xi}(t \wedge \sigma(w))=\Xi_{c, \xi}(t) \quad t \geqq 0$. Using (1.3), we can observe by the standard argument that for $p \geqq 1, T>0$ and $C>0$

$$
\begin{aligned}
& \mathbb{E}^{Q^{d}}\left[\max _{0 \leqq t}\left|\left(\Xi_{c^{\prime}, \xi^{\prime}}(t)-\xi^{\prime}\right)-\left(\Xi_{c, \xi}(t)-\xi\right)\right|^{2 p}\right] \\
& \quad \leqq \text { const. }\left\{\left|c^{\prime}-c\right|^{2 p}+\left|\xi^{\prime}-\xi\right|^{2 p}\right\} \quad 0 \leqq c, c^{\prime} \leqq C, \xi, \xi^{\prime} \in \mathbb{R}^{d-1}
\end{aligned}
$$

where the const. depends only on $d, p, T, C, K_{a}$ and $K_{b}$. From this estimate, we can prove the following:

Proposition $\mathbb{1}_{0} \mathbb{1}_{\text {. We }}$ can choose a nice modification of $\Xi_{c, \xi}(t)$ so that the following is satisfied:

(i ) $(c, \xi, t, w) \in[0, \infty) \times \mathbb{R}^{d-1} \times[0, \infty) \times \mathscr{W}_{0}^{d} \mapsto \Xi_{c, \xi}(t, w) \in \mathbb{R}^{d-1}$ is $\mathscr{B}[0$,

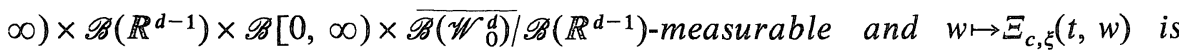
$\overline{\mathscr{B}_{t}\left(\mathscr{W}_{0}^{d}\right)}$-measurable for fixed $c, \xi$, $t$, where $\overline{\mathscr{B}\left(\mathscr{W}_{0}^{d}\right)}\left(\overline{\left.\mathscr{B}_{t}\left(\mathscr{W}_{0}^{d}\right)\right)}\right.$ is the completion of $\mathscr{B}\left(\mathscr{W}_{0}^{d}\right)\left(\right.$ resp. $\left.\mathscr{B}_{t}\left(\mathscr{W}_{0}^{d}\right)\right)$ by $Q^{d}$.

(ii) For fixed $w,(c, \xi, t) \mapsto \Xi_{c, \xi}(t, w)$ is continuous.

(iii) For every $c, \xi$, $t$ and $w, \Xi_{c, \xi}(t \wedge \sigma(w), w)=\Xi_{c, \xi}(t, w), \Xi_{c, \xi}(0, w)=\xi$ and $\Xi_{0, \xi}(t, w) \equiv \xi$.

Set $\mathbb{X}_{c, \xi}(t, w) \equiv\left(\Xi_{c, \xi}(t, w), c w^{d}(t)\right)$. Then $\mathbb{X}_{c, \xi} \in \mathscr{W}_{\partial}^{d}$ for each $c>0, \xi \in \mathbb{R}^{d-1}$ and $w \in \mathscr{W}_{0}^{d}, \mathbb{X}_{0, \xi} \equiv(\xi, 0)$ and a mapping $(c, \xi, w) \in[0, \infty) \times \mathbb{R}^{d-1} \times \mathscr{W}_{0}^{d} \mapsto \mathbb{X}_{c, \xi}(\cdot$,

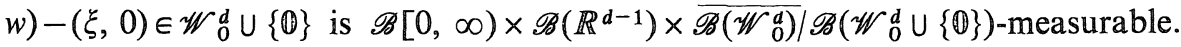

Moreover, by the estimates (1.3) and (1.5), we can also have

Proposition 1.2. Let $f: \mathbb{R}^{d} \rightarrow \mathbb{R}^{1}$ be bounded Lipschitz continuous. Then $\mathbb{I}_{f}^{i}(c, \xi, t, w)=\int_{0}^{t} f\left(\mathbb{X}_{c, \xi}(s, w)\right) d w^{i}(s) i=1, \ldots, d$ has a following nice modification:

(i ) $\quad(c, \xi, t, w) \in[0, \infty) \times \mathbb{R}^{d-1} \times[0, \infty) \times \mathscr{W}_{0}^{d} \mapsto \mathbb{I}_{f}^{i}(c, \xi, t, w) \in \mathbb{R}^{1}$ is $\mathscr{B}[0$, $\infty) \times \mathscr{B}\left(\mathbb{R}^{d-1}\right) \times \mathscr{B}[0, \infty) \times \overline{\mathscr{B}\left(\mathscr{W}_{0}^{d}\right)}$-measurable 
(ii) For fixed $w,(c, \xi, t) \mapsto \mathbb{I}_{f}^{i}(c, \xi, t, w)$ is continuous.

(iii) For every $c, \xi, t$ and $w, \mathbb{I}_{f}^{i}(c, \xi, t \wedge \sigma(w), w)=\mathbb{I}_{f}^{i}(c, \xi, t, w), \mathbb{I}_{f}^{i}(c, \xi$, $0, w)=0$ and $\mathbb{I}_{f}^{i}(0, \xi, t, w)=f(\xi, 0) w^{i}(t)$.

$\S \S 1.2 .3$. For each $n \geqq 1$ we define a left continuous function $[\cdot]_{n}$ on $[0, \infty)$ by

$$
[s]_{n}=\chi_{\{0\}}(s)+\sum_{k=0}^{\infty} k / 2^{n} \chi_{\left(k / 2^{n},(k+1) / 2^{n}\right]}(s) \quad s \geqq 0
$$

where $\chi_{I}$ is the indicator function of an interval $I$, and for a fixed $x, y \in \mathbb{R}^{d}$ and $\eta \geqq 0$ we consider the following $\mathrm{SDE}$ with respect to $Q^{d}$ :

$$
\begin{aligned}
& \mathbb{X}^{i}(s, w)=x^{i}+\sum_{j=1}^{d} \int_{0}^{s} \chi_{[\eta]_{n}+1 / 2^{n}-\eta \geqq \theta} a_{j}^{i}(y) d w^{j}(\theta) \\
&+\int_{0}^{s \wedge \sigma(w)} \chi_{[\eta]_{n}+1 / 2^{n}-\eta \geqq \theta} b^{i}(y) d \theta \\
&+\sum_{j=1}^{d} \int_{0}^{s} \chi_{[\eta]_{n}+1 / 2^{n}-\eta<\theta} a_{j}^{i}\left(\mathbb{X}\left(\left([\theta+\eta]_{n}-\eta\right)^{+}, w\right)\right) d w^{j}(\theta) \\
&+\int_{0}^{s \wedge \sigma(w)} \chi_{[\eta]_{n}+1 / 2^{n}-\eta<\theta} b^{i}\left(\mathbb{X}\left(\left([\theta+\eta]_{n}-\eta\right)^{+}, w\right)\right) d \theta \\
& i=1, \ldots, d .
\end{aligned}
$$

The solution of this $\mathrm{SDE}$ can be given by the following recursion formula:

$$
\mathbb{X}^{i}(s, w)=\left\{\begin{array}{c}
x^{i}+\sum_{j=1}^{d} a_{j}^{i}(y) w^{j}(s)+b^{i}(y) s \wedge \sigma(w) \quad i=1, \ldots, d \\
\quad \text { if } 0 \leqq s \leqq[\eta]_{n}+1 / 2^{n}-\eta \\
\mathbb{X}^{i}\left([\eta]_{n}+k / 2^{n}-\eta, w\right) \\
+\sum_{j=1}^{d} a_{j}^{i}\left(\mathbb{X}\left([\eta]_{n}+k / 2^{n}-\eta, w\right)\right)\left(w^{j}(s)-w^{j}\left([\eta]_{n}+k / 2^{n}-\eta\right)\right) \\
+b^{i}\left(\mathbb{X}\left([\eta]_{n}+k / 2^{n}-\eta, w\right)\right)\left(s \wedge \sigma(w)-\left([\eta]_{n}+k / 2^{n}-\eta\right) \wedge \sigma(w)\right) \\
\quad \text { if }[\eta]_{n}+k / 2^{n}-\eta<s \leqq[\eta]_{n}+(k+1) / 2^{n}-\eta \quad \text { and } k \geqq 1 .
\end{array}\right.
$$

If we denote it by $\mathbb{X}_{n}(x, y, \eta ; s, w)$, then the following is easily verified:

(i) For fixed $w$, a family $\left\{\mathbb{X}_{n}(x, y, \eta ; s, w)\right\}_{\eta \geqq 0}$ is equicontinuous in $(x, y, s) \in \mathbb{R}^{d} \times \mathbb{R}^{d} \times[0, \infty)$.

(ii) For fixed $x, y$ and $\eta, \mathbb{X}_{n}(x, y, \eta ; s, \cdot)$ is $\mathscr{B}_{s}\left(\mathscr{W}_{0}^{d}\right)$-measurable for each $s \geqq 0$ and $\mathbb{X}_{n}(x, y, \eta ; \cdot, \cdot)$ satisfies (1.6).

(iii) For fixed $x, y, s$ and $w, \eta \in[0, \infty) \mapsto \mathbb{X}_{n}(x, y, \eta ; s, w) \in \mathbb{R}^{d}$ is left continuous.

Moreover we have 
Proposition 1.3. For each $T>0$ there exists a constant $C$ depending only on $d,\|a\|_{\infty},\|b\|_{\infty}, K_{a}, K_{b}$ and $T$, and the following estimates hold:

$$
\sup _{\substack{x, y, z \in \mathbb{R}^{d} \\ \eta \geqq 0}} E^{Q^{d}}\left[\max _{0 \leqq s \leqq T}\left|X_{n}(x, y, \eta ; s, w)-X_{n}(x, z, \eta ; s, w)\right|^{2}\right] \leqq C\left(1 / 2^{n}\right)^{1 / 2}
$$

$$
\begin{gathered}
\text { (1.8) } \sup _{x \in \mathbb{R}^{d}} \sup _{\theta, \eta \geqq 0} E^{Q^{d}}\left[\left|X_{n}(x, x, \eta ; \theta, w)-X_{n}\left(x, x, \eta ;\left([\theta+\eta]_{n}-\eta\right)^{+}, w\right)\right|^{2}\right] \\
\leqq C\left(1 / 2^{n}\right)^{1 / 2}, \\
\text { (1.9) } \sup _{\eta \geqq 0} E^{Q^{d}}\left[\max _{0 \leqq s \leqq T}\left|X_{n}(x, x, \eta ; s, w)-x-\left(X_{1, \xi}(s, w)-(\xi, 0)\right)\right|^{2}\right] \\
\leqq C\left(\left(1 / 2^{n}\right)^{1 / 2}+|x-(\xi, 0)|^{2}\right) \quad x \in \mathbb{R}^{d}, \xi \in \mathbb{R}^{d-1} .
\end{gathered}
$$

Proof. For simplicity we denote $\mathbb{X}_{n}(x, y, \eta ; s, w)$ by $\mathbb{Z}_{n}(s, w)$ and $\mathbb{X}_{n}(x, z$, $\eta ; s, w)$ by $\mathbb{Z}_{n}(s, w)$. Since $\mathbb{Y}_{n}$ and $\mathbb{Z}_{n}$ satisfy (1.6), we observe by (1.3) and the Lipschitz continuity of $a$ and $b$ that for each $k \geqq 1$

$$
\begin{array}{r}
E^{Q^{d}}\left[\max \left\{\left|Y_{n}(s, w)-\mathbb{Z}_{n}(s, w)\right|^{2} ;[\eta]_{n}+1 / 2^{n}-\eta \leqq s \leqq[\eta]_{n}+(k+1) / 2^{n}-\eta\right\}\right] \\
\leq 3 E^{Q^{d}}\left[\left|Y_{n}\left([\eta]_{n}+1 / 2^{n}-\eta, w\right)-\mathbb{Z}_{n}\left([\eta]_{n}+1 / 2^{n}-\eta, w\right)\right|^{2}\right] \\
+ \text { const. }\left(1+k / 2^{n}\right) E^{Q^{d}}\left[\int_{\left([\eta]_{n}+1 / 2^{n}-\eta\right) \wedge \sigma(w)}^{\left([\eta]_{n}+(k+1) / 2^{n}-\eta\right) \wedge \sigma(w)} \mid Y_{n}\left([\theta+\eta]_{n}-\eta, w\right)\right. \\
\left.-\left.\mathbb{Z}_{n}\left([\theta+\eta]_{n}-\eta, w\right)\right|^{2} d \theta\right]
\end{array}
$$

where the const. depends only on $d, K_{a}$ and $K_{b}$. By setting

$$
\alpha_{k}=E^{Q^{d}}\left[\left|\mathbb{Y}_{n}\left([\eta]_{n}+k / 2^{n}-\eta, w\right)-\mathbb{Z}_{n}\left([\eta]_{n}+k / 2^{n}-\eta, w\right)\right|^{2}\right] \quad k \geqq 1,
$$

the above yields a series of inequalities of the Gronwall type:

$$
\alpha_{k+1} \leqq 3 \alpha_{1}+\text { const. }\left(1+k / 2^{n}\right) 1 / 2^{n} \sum_{l=1}^{k} \alpha_{l} \quad k \geqq 1 .
$$

Hence, we obtain

$$
\begin{aligned}
& E^{Q^{d}}\left[\operatorname { m a x } \left\{\left|\Psi_{n}(s, w)-\mathbb{Z}_{n}(s, w)\right|^{2} ;\right.\right. \\
& \left.\left.\quad[\eta]_{n}+1 / 2^{n}-\eta \leqq s \leqq[\eta]_{n}+(k+1) / 2^{n}-\eta\right\}\right] \\
& \quad \leqq 3 \alpha_{1} \frac{1+k / 2^{n}}{1+(k-1) / 2^{n}}\left\{1+1 / 2^{n} \text { const. }\left(1+(k-1) / 2^{n}\right)\right\}^{k} \quad k \geqq 1 .
\end{aligned}
$$

On the other hand, it is easy to see that

$$
E^{Q^{d}}\left[\max \left\{\left|\mathbb{Y}_{n}(s, w)-\mathbb{Z}_{n}(s, w)\right|^{2} ; 0 \leqq s \leqq[\eta]_{n}+1 / 2^{n}-\eta\right\}\right] \leqq \text { const. }\left(1 / 2^{n}\right)^{1 / 2}
$$

where the const. depends only on $d,\|a\|_{\infty}$ and $\|b\|_{\infty}$. Therefore, combining this with (1.10), we have (1.7).

Next, we show (1.8) and (1.9). To be simplified, we set $\mathbb{X}_{n}(s, w) \equiv \boldsymbol{X}_{n}(x, x, \eta$; 
$s, w)$ and $\mathbb{X}(s, w) \equiv \mathbb{X}_{1, \xi}(s, w)$. We note that $\mathbb{X}_{n}$ satisfies the following $\mathrm{SDE}$

$$
\begin{aligned}
\mathbb{X}^{i}(s, w)=x^{i} & +\sum_{j=1}^{d} \int_{0}^{s} a_{j}^{i}\left(\mathbb{X}\left(\left([\theta+\eta]_{n}-\eta\right)^{+}, w\right)\right) d w^{j}(\theta) \\
& +\int_{0}^{s \wedge \sigma(w)} b^{i}\left(\mathbb{X}\left(\left([\theta+\eta]_{n}-\eta\right)^{+}, w\right)\right) d \theta \quad i=1, \ldots, d .
\end{aligned}
$$

Noticing that $0 \leqq \theta-\left([\theta+\eta]_{n}-\eta\right)^{+} \leqq 1 / 2^{n}$, we can easily show (1.8). To prove (1.9), we define $\varepsilon_{n}^{i}(s)(i=1, \ldots, d)$ by

$$
\begin{aligned}
\varepsilon_{n}^{i}(s)= & \sum_{j=1}^{d} \int_{0}^{s}\left[a_{j}^{i}\left(\mathbb{X}_{n}\left(\left([\theta+\eta]_{n}-\eta\right)^{+}, w\right)\right)-a_{j}^{i}\left(\mathbb{X}_{n}(\theta, w)\right)\right] d w^{j}(\theta) \\
& +\int_{0}^{s \wedge \sigma(w)}\left[b^{i}\left(\mathbb{X}_{n}\left(\left([\theta+\eta]_{n}-\eta\right)^{+}, w\right)\right)-b^{i}\left(\mathbb{X}_{n}(\theta, w)\right)\right] d \theta
\end{aligned}
$$

By (1.8), it is easy to see that for each $T>0$

$$
\mathbb{E}^{Q^{d}}\left[\max _{0 \leqq s \leqq T}\left|\varepsilon_{n}(s)\right|^{2}\right] \leqq \text { const. }\left(1 / 2^{n}\right)^{1 / 2}
$$

where the const. depends only on $d, T,\|a\|_{\infty},\|b\|_{\infty}, K_{a}$ and $K_{b}$. And, by (1.4) and $(1.6)^{\prime}$, it holds that

$$
\begin{aligned}
& \mathbb{X}_{n}^{i}(s, w)-x^{i}-\left(\mathbb{X}^{i}(s, w)-\xi^{i}\right) \\
&= \varepsilon_{n}^{i}(s)+\sum_{j=1}^{d} \int_{0}^{s}\left[a_{j}^{i}\left(\mathbb{X}_{n}(\theta, w)\right)-a_{j}^{i}(\mathbb{X}(\theta, w))\right] d w^{j}(\theta) \\
& \quad+\int_{0}^{s \wedge \sigma(w)}\left[b^{i}\left[X_{n}(\theta, w)\right)-b^{i}(\mathbb{X}(\theta, w))\right] d \theta \quad i=1, \ldots, d,
\end{aligned}
$$

in which we set $\xi^{d}=0$ for convenience. Hence, by (1.3), (1.11) and the Lipschitz continuity of $a$ and $b$, we observe that for each $T>0$

$$
\begin{aligned}
& E^{Q^{d}}\left[\max _{0 \leqq s \leqq T}\left|\mathbb{X}_{n}(s, w)-x-(\mathbb{X}(s, w)-(\xi, 0))\right|^{2}\right] \\
& \leqq \text { const. }\left(\left(1 / 2^{n}\right)^{1 / 2}+|x-(\xi, 0)|^{2}\right) \\
& + \text { const. } \int_{0}^{T} E^{Q^{d}}\left[\left|\mathbb{X}_{n}(\theta, w)-x-(\mathbb{X}(\theta, w)-(\xi, 0))\right|^{2}\right] d \theta
\end{aligned}
$$

where the const. depends only on $d, T,\|a\|_{\infty},\|b\|_{\infty}, K_{a}$ and $K_{b}$. Thus (1.9) immediately follows from Gronwall's inequality.

Let $f: \mathbb{R}^{d} \rightarrow \mathbb{R}^{1}$ be a bounded Lipschitz continuous function with the Lipschitz constant $K_{f}$. For a fixed $x, y \in \mathbb{R}^{d}$ and $\eta \geqq 0$ we define $\mathbb{I}_{n}^{i}(x, y, \eta$; $t, w)$ by

$$
\mathbb{I}_{n}^{i}(x, y, \eta ; t, w)=\int_{0}^{t} f\left(\mathbb{X}_{n}\left(x, y, \eta ;\left([\theta+\eta]_{n}-\eta\right)^{+}, w\right)\right) d w^{i}(\theta) .
$$

Then, since $\mathbb{H}_{n}^{i}(x, y, \eta ; t, w)$ can be written as 


$$
\begin{aligned}
& \mathbb{Z}_{n}^{i}(x, y, \eta ; t, w) \\
& \quad=f(x) w^{i}\left(t \wedge\left([\eta]_{n}+1 / 2^{n}-\eta\right)\right) \\
& \quad+\sum_{k=1}^{\infty} f\left(\mathbb{X}_{n}\left(x, y, \eta ;[\eta]_{n}+k / 2^{n}-\eta, w\right)\right)\left(w^{i}\left(t \wedge\left([\eta]_{n}+(k+1) / 2^{n}-\eta\right)\right)\right. \\
& \left.\quad-w^{i}\left(t \wedge\left([\eta]_{n}+k / 2^{n}-\eta\right)\right)\right),
\end{aligned}
$$

it has the same properties on $x, y, \eta, t$ and $w$ as $X_{n}(x, y, \eta ; t, w)$. Moreover, as a corollary to Proposition 1.3, we have

Corollary 1.1. For each $T>0$ there exists a constant $C$ depending only on $d,\|a\|_{\infty},\|b\|_{\infty}, K_{a}, K_{b}, K_{f}$ and $T$, and it holds that

$$
\begin{aligned}
& \sup _{y \in \mathbb{R}^{d}, \eta \geqq 0} E^{Q^{d}}\left[\max _{0 \leqq t \leqq T}\left|\mathbb{H}_{n}^{i}(x, y, \eta ; t, w)-\mathbb{I}_{f}^{i}(1, \xi, t, w)\right|^{2}\right] \\
& \leqq C\left(\left(1 / 2^{n}\right)^{1 / 2}+|x-(\xi, 0)|^{2}\right) \quad x \in \mathbb{R}^{d}, \xi \in \mathbb{R}^{d-1}
\end{aligned}
$$

$\S \S 1.2 .4$. For $c \geqq 0$ and $w \in \mathscr{W}_{0}^{d}$ we define $T_{c} w \in \mathscr{W}_{0}^{d} \cup\{0\}$ by

$$
\left(\mathbb{T}_{c} w\right)(\cdot)= \begin{cases}c w\left(\cdot / c^{2}\right) \in \mathscr{W}_{0}^{d} & \text { if } c>0 \\ 0 & \text { if } c=0\end{cases}
$$

and we also define a measurable mapping $\Phi: \mathbb{R}^{d-1} \times \mathscr{W}_{0}^{d} \cup\{\mathbb{O}\} \rightarrow \mathscr{W}_{0}^{d} \cup\{\mathbb{O}\}$ as follows:

$$
\Phi[\xi, w](\cdot)= \begin{cases}\mathbb{X}_{1, \xi}(\cdot, w)-(\xi, 0) \in \mathscr{W}_{0}^{d} & \text { if } w \in \mathscr{W}_{0}^{d} \\ 0 & \text { if } w=\mathbb{0}\end{cases}
$$

Then, by (1.1) it is easy to see that for each $c>0$

$$
Q^{d, \pm}\left\{w ; T_{c} w \in *\right\}=c Q^{d, \pm}(*) \quad * \in \mathscr{B}\left(\mathscr{W}_{0}^{d, \pm}\right),
$$

and, by using this we can verify that for each $c>0$ and $\xi \in \mathbb{R}^{d-1}$

$$
\mathbb{X}_{c, \xi}\left(\cdot / c^{2}, w\right)=\mathbb{X}_{1, \xi}\left(\cdot, \mathbb{T}_{c} w\right) \quad \text { for a.a. } w\left(Q^{d}\right) .
$$

Hence, as to $\Phi$, it holds that for each $c \geqq 0$ and $\xi \in \mathbb{R}^{d-1}$

$$
\Phi\left[\xi, T_{c} w\right]\left(c^{2} \sigma(w)\right)=\mathbb{X}_{c, \xi}(\sigma(w), w)-(\xi, 0) \quad \text { for a.a. } \quad w\left(Q^{d}\right) .
$$

In view of (1.4), we have the following estimate: For $c_{0}>0$

$$
\left.\sup _{\substack{\xi \in \mathbb{R}^{d}-1 \\ 0 \leqq c \leqq c_{0}}} E^{Q^{d}} \underset{0 \leqq s \leqq t}{ }\left|\mathbb{X}_{c, \xi}(s)-(\xi, 0)\right|^{2}\right] \leqq \text { const. }(1+t) \sqrt{t}
$$

where the const. depends only on $d, c_{0},\|a\|_{\infty}$ and $\|b\|_{\infty}$. Using this estimate, we see easily that for some constant $C$ depending only on $d,\|a\|_{\infty}$ and $\|b\|_{\infty}$

$$
\sup _{\xi \in \mathbb{R}^{d}-1} \mathbb{E}^{Q^{d}}\left[|\Phi[\xi, w](\sigma(w))|^{2} ; \sigma(w) \leqq \eta\right] \leqq C(1+\eta) \sqrt{\eta} \eta \geqq 0
$$


(1.16) $\sup _{\xi \in \mathbb{R}^{d-1}} \mathbb{E}^{Q^{d}}\left[|\Phi[\xi, w](\sigma(w))| ; \eta \leqq \sigma(w) \leqq \eta^{\prime}\right] \leqq C\left(\left(1+\eta^{\prime}\right)\left(\sqrt{\eta^{\prime} / \eta}-1\right)\right)^{1 / 2}$

$$
0<\eta \leqq \eta^{\prime} \text {. }
$$

Further we have

Proposition 1.4. As $\varepsilon \downarrow 0$, the following holds:

$$
\begin{array}{ll}
\sup _{\substack{\xi \in \mathbb{R}^{d-1} \\
0 \leqq c^{ \pm} \leqq 1}} \mid c^{ \pm} \mathbb{E}^{Q^{d, \pm}}\left[\Phi[\xi, w]^{\prime}(\sigma(w)) ; \varepsilon\left(c^{ \pm}\right)^{2}<\sigma(w) \leqq\left(c^{ \pm}\right)^{2}\right] \\
& -\left(\left(-c^{ \pm}\right) E^{Q^{d, \pm}}\left[\int_{0}^{1} a_{d}^{\prime}\left(X_{c^{ \pm}, \xi}(s)\right) d w^{d}(s): \sigma(w)>1\right]\right. \\
& +\left(c^{ \pm}\right)^{2} E^{Q^{d, \pm}}\left[\int_{0}^{\sigma(w)} b^{\prime}\left(X_{c^{ \pm}, \xi}(s)\right) d s ; \sigma(w) \leqq 1\right] \\
& \pm c^{ \pm} a_{d}^{\prime}(\xi, 0) \mid \longrightarrow 0,
\end{array}
$$

$$
\sup _{\substack{\xi \in \mathbb{R}^{d-1} \\ 0<c^{ \pm} \leqq 1}}\left|\mathbb{E}^{Q^{d, \pm}}\left[\Phi[\xi, w]^{\prime}(\sigma(w)) ; \varepsilon\left(c^{ \pm}\right)^{2}<\sigma(w) \leqq \varepsilon\right]\right| \longrightarrow 0 .
$$

Here $x^{\prime}$ denotes $\left(x^{1}, \ldots, x^{d-1}\right)$ for $x=\left(x^{1}, \ldots, x^{d}\right) \in \mathbb{R}^{d}$.

Proof. From (1.12) and (1.13), we see

$$
\begin{gathered}
c^{ \pm} E^{Q^{d, \pm}}\left[\Phi[\xi, w]^{\prime}(\sigma(w)) ; \varepsilon\left(c^{ \pm}\right)^{2}<\sigma(w) \leqq\left(c^{ \pm}\right)^{2}\right] \\
=E^{Q^{d, \pm}}\left[\Xi_{c^{ \pm}, \xi}(\sigma(w), w)-\xi ; \varepsilon<\sigma(w) \leqq 1\right] .
\end{gathered}
$$

Since $\Xi_{c^{ \pm}, \xi}$ satisfies (1.4), using the martingale property of stochastic integrals with respect to $Q^{d}$, we observe that the right hand side in the above is equal to

$$
\begin{aligned}
& \left(c^{ \pm}\right)^{2} E^{Q^{d, \pm}}\left[\int_{0}^{\sigma(w)} b^{\prime}\left(\mathbb{X}_{c^{ \pm}, \xi}(s)\right) d s ; \sigma(w) \leqq 1\right] \\
& -c^{ \pm} E^{Q^{d, \pm}}\left[\int_{0}^{1} a_{d}^{\prime}\left(\mathbb{X}_{c^{ \pm}, \xi}(s)\right) d w^{d}(s) ; \sigma(w)>1\right] \pm c^{ \pm} a_{d}^{\prime}(\xi, 0) \\
& -\left(c^{ \pm}\right)^{2} E^{Q^{d, \pm}}\left[\int_{0}^{\sigma(w)} b^{\prime}\left(\mathbb{X}_{c^{ \pm}, \xi}(s)\right) d s ; \sigma(w) \leqq \varepsilon\right] \\
& +c^{ \pm} E^{Q^{d, \pm}}\left[\int_{0}^{\varepsilon}\left(a_{d}^{\prime}\left(\mathbb{X}_{c^{ \pm}, \xi}(s)\right)-a_{d}^{\prime}(\xi, 0)\right) d w^{d}(s) ; \sigma(w)>\varepsilon\right] .
\end{aligned}
$$

Here we have used the fact that $\mathbb{E}^{Q^{d, \pm}}\left[w^{d}(\varepsilon) ; \sigma(w)>\varepsilon\right]= \pm 1$. Therefore, by noting that $E^{Q^{d, \pm}}[\varepsilon \wedge \sigma]=4 \sqrt{\varepsilon / 2 \pi}$ and $Q^{d, \pm}(\sigma>\varepsilon)=\sqrt{2 / \pi \varepsilon}$, and by (1.14), (1.17) follows immediately. By tracing the similar argument as above, we can also show (1.18). [

\section{§2。 Uniquinemess II}

Let $a, b, \tau, \beta$ and $\mu$ satisfy $(0.2)^{\prime},(0.3),(0.4),(0.5)^{\prime}$ and (0.6). Namely, 
$a, \tau: \mathbb{R}^{d} \rightarrow \mathbb{R}^{d} \otimes \mathbb{R}^{d}$ and $b, \beta: \mathbb{R}^{d} \rightarrow \mathbb{R}^{d}$ are bounded Lipschitz continuous functions satisfying

$$
\begin{aligned}
& a_{j}^{d}=\delta_{j}^{d} \quad j=1, \ldots, d, \quad b^{d}=0 \\
& \tau_{j}^{d}=0 \quad j=1, \ldots, d \\
& \sup _{\xi \in R^{d}-1}\left|\beta^{d}(\xi, 0)\right| \leqq 1 .
\end{aligned}
$$

Our aim of this section is to show the uniqueness of $\mathbb{S}_{\lambda}\left[a, b, \tau, \beta, \delta_{0}\right]$ for any probability measure $\lambda(d x)$ on $\mathbb{R}^{d}$.

Let $\lambda(d x)$ be a probability measure on $\mathbb{R}^{d}$ and let $[X(t), B(t), M(t)]$ be an element of $\boldsymbol{S}_{\lambda}\left[a, b, \tau, \beta, \delta_{0}\right]$ defined on a filtered probability space $(\Omega, \mathscr{F}, \mathbb{P}$, $\left.\mathscr{F}_{t}\right)$. We set $\sigma_{0}=\sigma_{0}(X)=\inf \left\{t \geqq 0 ; X^{d}(t)=0\right\}$. Then

$$
\sigma_{0}=\inf \left\{t \geqq 0 ; X^{d}(0)+B^{d}(t)=0\right\}
$$

and since $L_{t \wedge \sigma_{0}}^{0}\left(X^{d}\right)=0$ for any $t \geqq 0$, it is easy to see that $M\left(t \wedge \sigma_{0}\right)=0$ for any $t \geqq 0$. Hence, for $i=1, \ldots, d$

$$
X^{i}\left(t \wedge \sigma_{0}\right)=X^{i}(0)+\sum_{j=1}^{d} \int_{0}^{t} a_{j}^{i}\left(X\left(s \wedge \sigma_{0}\right)\right) d B^{j}\left(s \wedge \sigma_{0}\right)+\int_{0}^{t} b^{i}\left(X\left(s \wedge . \sigma_{0}\right)\right) d s \wedge \sigma_{0},
$$

which, together with (2.4), implies that

$$
P\left(X\left(\cdot \wedge \sigma_{0}\right) \in *\right)=\int_{\mathbb{R}^{d}} \stackrel{\circ}{P}^{x}(*) \lambda(d x) .
$$

Here, $\stackrel{P}{P}^{x}$ denotes a probability measure on $\mathscr{W}^{d}$ introduced in $\S \S 1.1$ for the functions $a$ and $b$. Next we define

$$
\bar{X}(t)=X\left(t+\sigma_{0}\right), \quad \bar{B}(t)=B\left(t+\sigma_{0}\right)-B\left(\sigma_{0}\right), \quad \bar{M}(t)=M\left(t+\sigma_{0}\right), \quad \overline{\mathscr{F}}_{t}=\mathscr{F}_{t+\sigma_{0}}
$$

where $\sigma_{0}$ is finite a.s. $(P)$ from (2.4). Clearly $[\bar{X}(t), \bar{B}(t), \bar{M}(t)] \in \mathbb{S}_{P\left(x\left(\sigma_{0}\right) \in *\right)}[a$, $\left.b, \tau, \beta, \delta_{0}\right]$ and further, with probability one, it belongs to $\mathbb{S}_{\delta_{\left(\Xi\left(\sigma_{0}\right), 0\right)}}[a, b, \tau$, $\left.\beta, \delta_{0}\right]$ with respect to $P\left(\cdot \mid \overline{\mathscr{F}}_{0}\right)$. Now, if we suppose that for each $\xi \in \mathbb{R}^{d-1}$

$$
\text { the uniqueness holds for } \mathbb{S}_{\delta_{(\xi, 0)}}\left[a, b, \tau, \beta, \delta_{0}\right]
$$

and we denote by $P_{\xi}$ the unique probability law of $X(\cdot)$, then it follows from the above that

$$
P\left(\bar{X} \in * \mid \overline{\mathscr{F}}_{0}\right)=P_{\Xi\left(\sigma_{0}\right)}(*) \text { a.s. }(P) .
$$

Therefore, putting (2.5) and this together, we have that for $0<t_{1}<\cdots<t_{n}$ 


$$
\begin{aligned}
& P\left(X\left(t_{1}\right) \in *_{1}, \ldots, X\left(t_{n}\right) \in *_{n}\right) \\
& =\sum_{i=1}^{n} \int_{\mathbb{R}^{d}} \stackrel{\circ}{E}^{x}\left[P_{w^{\prime}\left(\sigma_{0}\right)}\left(w\left(s_{i}\right) \in *_{i}, \ldots, w\left(s_{n}\right) \in *_{n}\right)\left|s_{i}=t_{i}-\sigma_{0}, \ldots, s_{n}=t_{n}-\sigma_{0}\right|\right. \\
& \left.\quad ; w\left(t_{1}\right) \in *_{1}, \ldots, w\left(t_{i-1}\right) \in *_{i-1}, t_{i-1} \leqq \sigma_{0}<t_{i}\right] \lambda(d x) \\
& \quad+\int_{\mathbb{R}^{d}} \stackrel{\circ}{P}^{x}\left(w\left(t_{1}\right) \in *_{1}, \ldots, w\left(t_{n}\right) \in *_{n}, t_{n} \leqq \sigma_{0}\right) \lambda(d x)
\end{aligned}
$$

and thus, the uniqueness for $\mathbb{S}_{\lambda}\left[a, b, \tau, \beta, \delta_{0}\right]$ follows. Consequently, we have seen that if we prove (2.6) for each $\xi \in \mathbb{R}^{d-1}$, then our aim in this section is attained. In the following, we shall present four propositions and we shall prove (2.6).

We fix $\xi_{0} \in \mathbb{R}^{d-1}$, and let $[X(t), B(t), M(t)]$ be an element of $\mathbb{S}_{\delta_{\left(\xi_{0}, 0\right)}}[a, b$, $\left.\tau, \beta, \delta_{0}\right]$ defined on a filtered probability space $\left(\Omega, \mathscr{F}, P, \mathscr{F}_{t}\right)$. We may assume that $\mathscr{F}=\vee_{t>0} \mathscr{F}_{t}$ and $X(0)=\left(\xi_{0}, 0\right)$. By $(2.1)$ and $(2.2)$, if we denote $\tau_{j}^{i}(\cdot, 0)$ and $\beta^{i}(\cdot, 0)$ by $\tau_{j}^{i}(\cdot)$ and $\beta^{i}(\cdot)$ respectively, then the $\operatorname{SDE}(0.1)^{\prime}$ is rewritten as

$$
\begin{gathered}
X^{i}(t)=\xi_{0}^{i}+\sum_{j=1}^{d} \int_{0}^{t} a_{j}^{i}(X(s)) d B^{j}(s)+\int_{0}^{t} b^{i}(X(s)) d s \\
+\sum_{j=1}^{d} \int_{0}^{t} \tau_{j}^{i}(\Xi(s)) d M^{j}(s)+\int_{0}^{t} \beta^{i}(\Xi(s)) d L_{s}^{0}\left(X^{d}\right) \quad i=1, \ldots, d-1 \\
X^{d}(t)=B^{d}(t)+\int_{0}^{t} \beta^{d}(\Xi(s)) d L_{s}^{0}\left(X^{d}\right)
\end{gathered}
$$

where $\Xi(t)=\left(X^{1}(t), \ldots, X^{d-1}(t)\right)$. Applying Itô's formula to (2.8), we observe

$$
\left|X^{d}(t)\right|=\int_{0}^{t} \operatorname{sgn}\left(X^{d}(s)\right) d B^{d}(s)+L_{t}^{0}\left(X^{d}\right) .
$$

This implies that $\left[\left|X^{d}(t)\right|, L_{t}^{0}\left(X^{d}\right)\right]$ is a solution of the Skorohod equation and hence, by setting $\widetilde{B}^{d}(t) \equiv \int_{0}^{t} \operatorname{sgn}\left(X^{d}(s)\right) d B^{d}(s)$, it follows that

$$
\begin{aligned}
& L_{t}^{0}\left(X^{d}\right)=-\min _{0 \leqq s \leqq t} \widetilde{B}^{d}(s), \\
& \left|X^{d}(t)\right|=\widetilde{B}^{d}(t)-\min _{0 \leqq s \leqq t} \widetilde{B}^{d}(s)
\end{aligned}
$$

(cf. [5] or Lemma III-4.2 in [2]). Therefore, noting that $\widetilde{B}^{d}(t)$ is also an $\mathscr{F}_{t}-B M_{0}^{1}$, we see that $L_{\infty}^{0}\left(X^{d}\right)=\infty$ a.s. $(P)$, and if we set $A(t) \equiv \inf \left\{s ; L_{s}^{0}\left(X^{d}\right)>t\right\}$, then $A(t)<\infty$ for any $t \geqq 0$ and $t \mapsto A(t)$ is strictly increasing right continuous with $A(0)=0$ and $A(\infty)=\infty$ a.s. $(P)$. Moreover we see from the above that with probability one

$$
\begin{aligned}
& X^{d}(A(t))=0 \quad(t \geqq 0), \quad X^{d}(A(t-))=0 \quad(t>0) \\
& \left|X^{d}(u)\right|>0 \quad \text { if } \quad A(t-)<u<A(t) \quad \text { and } \quad t>0 .
\end{aligned}
$$


Now, from this and the fact:

$$
X^{d}((s+A(t-)) \wedge A(t))=B^{d}((s+A(t-)) \wedge A(t))-B^{d}(A(t-)) \quad s \geqq 0, \quad t>0,
$$

we define $\mathscr{F}_{A(t)}$-point processes $p$ and $q$ on $\mathscr{W}_{0}^{d}$ as follows:

$$
\begin{aligned}
& \mathbb{D}_{p}=\mathbb{D}_{q}=\mathbb{D} \equiv\{t>0 ; A(t)>A(t-)\}, \\
& p[t](\cdot) \equiv B((\cdot+A(t-)) \wedge A(t))-B(A(t-)) \quad t \in \mathbb{D}_{p}, \\
& q[t](\cdot) \equiv X((\cdot+A(t-)) \wedge A(t))-X(A(t-)) \quad t \in \mathbb{D}_{q} .
\end{aligned}
$$

We also put $\xi(t) \equiv \Xi(A(t))(t \geqq 0)$ and $c^{ \pm}(\xi) \equiv\left\{1 \pm \beta^{d}(\xi)\right\} / 2 \quad\left(\xi \in \mathbb{R}^{d-1}\right)$. Then $\xi(t)$ is an $\mathscr{F}_{A(t)}$-adapted $(d-1)$-dimensional right continuous process and $c^{ \pm}(\xi)$ is nonnegative from (2.3).

Firstly, as to an $\mathscr{F}_{A(t)}$-point process $p$ on $\mathscr{W}_{0}^{d}$, we state the following. The idea of the proof is due to $\mathrm{S}$. Watanabe.

Proposition 2.1. An $\mathscr{F}_{A(t)}$-point process $p$ on $\mathscr{W}_{0}^{d}$ is of class $Q L$ with compensator

$$
\widehat{N}_{p}(d t d w)=c^{+}(\xi(t-)) d t Q^{d,+}\left(d w \cap \mathscr{W}_{0}^{d,+}\right)+c^{-}(\xi(t-)) d t Q^{d,-}\left(d w \cap \mathscr{W}_{0}^{d,-}\right) .
$$

Proof. It is sufficient to show that for $t^{\prime}>t$ and $\Gamma \in \mathscr{B}\left(\mathscr{W}_{0}^{d, \pm}\right)$ with $Q^{d, \pm}(\Gamma)$ $<+\infty$,

$$
E\left[\#\left\{s \in\left(t, t^{\prime}\right] ; p[s] \in \Gamma\right\} \mid \mathscr{F}_{A(t)}\right]=E\left[\int_{t}^{t^{\prime}} c^{ \pm}(\xi(s-)) d s \mid \mathscr{F}_{A(t)}\right] Q^{d, \pm}(\Gamma) .
$$

To do this, we set

$$
\begin{aligned}
& \sigma_{ \pm}(t)=\int_{0}^{t} \chi_{\boldsymbol{R}_{ \pm}}^{\circ}\left(X^{d}(s)\right) d s, \\
& N_{ \pm}^{i}(t)=\int_{0}^{t} \chi_{\boldsymbol{R}_{ \pm}}\left(X^{d}(s)\right) d B^{i}(s) \quad i=1, \ldots, d-1, \quad N_{ \pm}^{d}(t)= \pm \int_{0}^{t} \chi_{\mathbb{R}_{ \pm}}\left(X^{d}(s)\right) d B^{d}(s),
\end{aligned}
$$

where $\stackrel{\circ}{\mathbb{R}}_{+}=(0, \infty)$ and $\stackrel{\circ}{\mathbb{R}}_{-}=(-\infty, 0)$. By applying Itô's formula to $(2.8)$, we first note that

$$
X_{ \pm}^{d}(t)=N_{ \pm}^{d}(t)+\int_{0}^{t} c^{ \pm}(\Xi(s)) d L_{s}^{0}\left(X^{d}\right)
$$

where $X_{+}^{d}(t)=X^{d}(t) \vee 0$ and $X_{-}^{d}(t)=\left(-X^{d}(t)\right) \vee 0$. Similarly as $\left|X^{d}(t)\right|$, this expression implies that

$$
\left\{\begin{array}{l}
\int_{0}^{t} c^{ \pm}(\Xi(s)) d L_{s}^{o}\left(X^{d}\right)=-\min _{0 \leqq s \leqq t} N_{ \pm}^{d}(s) \\
X_{ \pm}^{d}(t)=N_{ \pm}^{d}(t)-\min _{0 \leqq s \leqq t} N_{ \pm}^{d}(s) .
\end{array}\right.
$$


Noting that $\lim _{t \uparrow \infty} N_{ \pm}^{i}(t)$ exists and is finite a.s. on $\left[\sigma_{ \pm}(\infty)=\left\langle N_{ \pm}^{i}\right\rangle(\infty)<+\infty\right]$, we define $\bar{B}_{ \pm}^{i}(t) i=1, \ldots, d$ as

$$
\bar{B}_{ \pm}^{i}(t)= \begin{cases}\lim _{t \uparrow \infty} N_{ \pm}^{i}(t) & \text { if } t \geqq \sigma_{ \pm}(\infty) \\ N_{ \pm}^{i}\left(\tau_{ \pm}(t)\right) & \text { if } t<\sigma_{ \pm}(\infty)\end{cases}
$$

where $\tau_{ \pm}(t)=\inf \left\{s ; \sigma_{ \pm}(s)>t\right\}$. Then $\left[\bar{B}_{ \pm}^{1}, \ldots, \bar{B}_{ \pm}^{d}\right]$ is a $d$-dimensional Brownian motion up to time $\sigma_{ \pm}(\infty)$ and it is clear that

$$
\bar{B}_{ \pm}^{i}\left(\sigma_{ \pm}(t)\right)=N_{ \pm}^{i}(t) \quad i=1, \ldots, d .
$$

Now, like as $p[t]$, we define $\bar{p}_{ \pm}[t] \in \mathscr{W}_{0}^{d,+} \cup\{\mathbb{O}\}$ for $t>0$ as follows:

$$
\begin{aligned}
& l_{ \pm}(t)=-\min _{0 \leqq s \leqq t} \bar{B}_{ \pm}^{d}(s), \quad\left(l_{ \pm}\right)^{-1}(t)=\inf \left\{s ; l_{ \pm}(s)>i\right\} \\
& \bar{p}_{ \pm}[t](\cdot)=\bar{B}_{ \pm}\left(\left(\cdot+\left(l_{ \pm}\right)^{-1}(t-)\right) \wedge\left(l_{ \pm}\right)^{-1}(t)\right)-\bar{B}_{ \pm}\left(\left(l_{ \pm}\right)^{-1}(t-)\right) .
\end{aligned}
$$

Then, by the general theory of time change, the following holds: For $t^{\prime}>t$ and $\Gamma \in \mathscr{B}\left(\mathscr{W}_{0}^{d,+}\right)$ with $Q^{d,+}(\Gamma)<+\infty$

$$
\begin{aligned}
& \mathbb{E}\left[\#\left\{s \in\left(t, t^{\prime}\right] ; \bar{p}_{ \pm}[s] \in \Gamma\right\} \mid \overline{\mathscr{F}} \frac{ \pm}{\left(l_{ \pm}\right)^{-1}(t)}\right] \\
& \quad=\mathbb{E}\left[t^{\prime} \wedge l_{ \pm}(\infty)-t \wedge l_{ \pm}(\infty) \mid \overline{\mathscr{F}}\left(l_{ \pm}\right)^{-1}(t)\right] Q^{d,+}(\Gamma)
\end{aligned}
$$

where $\overline{\mathscr{F}}_{t}^{ \pm}=\mathscr{F}_{\tau_{ \pm}(t)}$.

On the other hand, we can observe that for $t>0$ and $\Gamma \in \mathscr{B}\left(\mathscr{W}_{0}^{d, \pm}\right)$

$$
\begin{aligned}
& \#\{s \in(0, t] ; p[s] \in \Gamma\} \\
& \quad=\#\left\{s \in\left(0, l_{ \pm}\left(\sigma_{ \pm}(A(t))\right)\right] ;\left(\bar{p}_{ \pm}[s]^{1}, \ldots, \bar{p}_{ \pm}[s]^{d-1}, \pm \bar{p}_{ \pm}[s]^{d}\right) \in \Gamma\right\} .
\end{aligned}
$$

Indeed, this is shown in the following: Firstly, by virtue of (2.11), we note that (2.10) becomes

$$
\left\{\begin{array}{l}
l_{ \pm}\left(\sigma_{ \pm}(t)\right)=\int_{0}^{t} c^{ \pm}(\Xi(s)) d L_{s}^{0}\left(X^{d}\right) \\
X_{ \pm}^{d}(t)=\bar{B}_{ \pm}^{d}\left(\sigma_{ \pm}(t)\right)+l_{ \pm}\left(\sigma_{ \pm}(t)\right) .
\end{array}\right.
$$

From this, it follows immediately that

$$
l_{ \pm}\left(\sigma_{ \pm}(A(t))\right)=\int_{0}^{i} c^{ \pm}(\xi(s)) d s
$$

Now, let $t>0$ be such as $p[t] \in \mathscr{W}_{0}^{d, \pm}$. Then, since $X^{d}(u) \in \mathbb{R}_{ \pm}^{\circ}$ for any $u$; $A(t-)<u<A(t)$, it follows from the definition of $\sigma_{ \pm}$and $N_{ \pm}^{i}$ that

$$
N_{ \pm}^{i}(u+A(t-))-N_{ \pm}^{i}(A(t-))= \begin{cases}p[t]^{i}(u) & \text { if } i \in\{1, \ldots, d-1\} \\ \pm p[t]^{d}(u) & \text { if } i=d\end{cases}
$$




$$
\sigma_{ \pm}(u+A(t-))=\sigma_{ \pm}(A(t-))+u
$$

for $0 \leqq u \leqq A(t)-A(t-)$. Hence, these, together with (2.11), imply that for $0 \leqq u \leqq A(t)-A(t-)$

$$
\bar{B}_{ \pm}^{i}\left(u+\sigma_{ \pm}(A(t-))\right)-\bar{B}_{ \pm}^{i}\left(\sigma_{ \pm}(A(t-))\right)= \begin{cases}p[t]^{i}(u) & \text { if } i \in\{1, \ldots, d-1\} \\ \pm p[t]^{d}(u) & \text { if } i=d\end{cases}
$$

On the other hand, if we notic that $\bar{B}_{ \pm}^{d}+l_{ \pm}$is a 1-dimensional reflecting Brownian motion up to time $\sigma_{ \pm}(\infty)$, then, by $(2.10)^{\prime}$ we easily verify that $\left(l_{ \pm}\right)^{-1}$ $\left(l_{ \pm}\left(\sigma_{ \pm}(A(t))\right)-\right)=\sigma_{ \pm}(A(t-))$ and $\left(l_{ \pm}\right)^{-1}\left(l_{ \pm}\left(\sigma_{ \pm}(A(t))\right)\right)=\sigma_{ \pm}(A(t))$. Therefore, combining this and the above, we have

$$
p[t]=\left(\bar{p}_{ \pm}[s]^{1}, \ldots, \bar{p}_{ \pm}[s]^{d-1}, \pm \bar{p}_{ \pm}[s]^{d}\right)
$$

where $s=l_{ \pm}\left(\sigma_{ \pm}(A(t))\right)$, which, as to (2.13), says that the left hand side is less than or equal to the right one. Next we show the opposite sign of inequality. For this, we fix $t>0$ and let $s>0$ be such as $0<s \leqq l_{ \pm}\left(\sigma_{ \pm}(A(t))\right)$ and $\bar{p}_{ \pm}[s] \in \mathscr{W}_{0}^{d,+}$. We take $t_{0}=\inf \left\{t^{\prime} ; s=l_{ \pm}\left(\sigma_{ \pm}\left(A\left(t^{\prime}\right)\right)\right)\right\}$. Then, by noting that

$$
\left(l_{ \pm}\right)^{-1}\left(l_{ \pm}\left(\sigma_{ \pm}(A(u))\right)\right)=\sigma_{ \pm}(A(u)) \quad \text { for any } u \geqq 0
$$

we observe that

$$
\begin{aligned}
& \left(l_{ \pm}\right)^{-1}(s)=\left(l_{ \pm}\right)^{-1}\left(l_{ \pm}\left(\sigma_{ \pm}\left(A\left(t_{0}\right)\right)\right)\right)=\sigma_{ \pm}\left(A\left(t_{0}\right)\right) \\
& \left(l_{ \pm}\right)^{-1}(s-)=\lim _{t^{\prime} \uparrow t_{0}}\left(l_{ \pm}\right)^{-1}\left(l_{ \pm}\left(\sigma_{ \pm}\left(A\left(t^{\prime}\right)\right)\right)\right)=\lim _{t^{\prime} \uparrow t_{0}} \sigma_{ \pm}\left(A\left(t^{\prime}\right)\right)=\sigma_{ \pm}\left(A\left(t_{0}-\right)\right) .
\end{aligned}
$$

Since $\left(l_{ \pm}\right)^{-1}(s)>\left(l_{ \pm}\right)^{-1}(s-)$, this implies $A\left(t_{0}\right)>A\left(t_{0}-\right)$, that is, $p\left[t_{0}\right] \in \mathscr{W}_{0}^{d, \pm}$. Thus, as we saw in the above, it follows that

$$
p\left[t_{0}\right]=\left(\bar{p}_{ \pm}[s]^{1}, \ldots, \bar{p}_{ \pm}[s]^{d-1}, \pm \bar{p}_{ \pm}[s]^{d}\right) .
$$

Therefore, as to (2.13), we obtain the another inequality and consequently, the proof of (2.13) is complete.

Finally we prove (2.9): We denote $\overline{\mathscr{F}}_{\left(l_{ \pm}\right)^{-1}\left(l_{ \pm}\left(\sigma_{ \pm}(A(t))\right)\right)}$ by $\mathscr{G}_{t}$ for simplicity. Applying the optional sampling theorem, we conclude by (2.12), (2.13) and (2.14) that for $t^{\prime}>t$ and $\Gamma \in \mathscr{B}\left(\mathscr{W}_{0}^{d, \pm}\right)$ with $Q^{d, \pm}(\Gamma)<+\infty$

$$
E\left[\#\left\{s \in\left(t, t^{\prime}\right] ; p[s] \in \Gamma\right\} \mid \mathscr{G}_{t}\right]=E\left[\int_{t}^{t^{\prime}} c^{ \pm}(\xi(s-)) d s \mid \mathscr{G}_{\iota}\right] Q^{d, \pm}(\Gamma) .
$$

Thus. (2.9) follows immediately by noting that $\mathscr{G}_{t} \supset \mathscr{F}_{A(t)}$.

灆

Secondly, as to an $\mathscr{F}_{A(t)}$-point process $q$ on $\mathscr{W}_{0}^{d}$, we present the following:

Proposition 2.2. With probability one, it holds that for any $t \in \mathbb{D}$ 


$$
q[t](\cdot)=\Phi[\xi(t-), p[t]](\cdot) .
$$

Here $\Phi[\xi, w]$ is introduced in $\$ § 1.2 .4$.

To prove this proposition, we present the following lemma: For each $n \geqq 1$, let $[\cdot]_{n}$ be a left continuous function on $[0, \infty)$ defined in $\S \S 1.2 .3$ and let $\left[X_{n}(t)\right.$ $\left.=\left(X_{n}^{1}(t), \ldots, X_{n}^{d}(t)\right)\right]$ denote a unique solution of the following SDE:

$$
\begin{aligned}
& Y^{i}(t)=\xi_{0}^{i}+\sum_{j=1}^{d} \int_{0}^{t} a_{j}^{i}\left(Y\left([s]_{n}\right)\right) d B^{j}(s)+\int_{0}^{t} b^{i}\left(Y\left([s]_{n}\right)\right) d s \\
& +\sum_{j=1}^{d} \int_{0}^{t} \tau_{j}^{i}\left(Y^{\prime}\left([s]_{n}\right)\right) d M^{j}(s)+\int_{0}^{t} \beta^{i}\left(Y^{\prime}\left([s]_{n}\right)\right) d L_{s}^{0}\left(X^{d}\right) \quad i=1, \ldots, d
\end{aligned}
$$

where $Y^{\prime}(t)=\left(Y^{1}(t), \ldots, Y^{d-1}(t)\right)$ and $\xi_{0}^{d}=0$ for convenience. Then we have

Lemma 2.耳. For each $T>0$ and $p>0$

$$
\begin{aligned}
& \lim _{n \uparrow \infty} E\left[\sup _{0 \leqq t \leqq T}\left|X_{n}(t)-X_{n}\left([t]_{n}\right)\right|^{p}\right]=0, \\
& \lim _{n \uparrow \infty} E\left[\max _{0 \leqq t \leqq T}\left|X(t)-X_{n}(t)\right|^{p}\right]=0 .
\end{aligned}
$$

The proof of this lemma is carried out in the standard way, and so we omit it.

Proof of Proposition 2.2. For simplicity we denote $L_{t}^{0}\left(X^{d}\right)$ by $L(t)$. It is enough to prove that for each $\varepsilon>0$ and $T>0$

$$
E\left[\sum_{0<s<L(T)} \chi_{A(s)-A(s-)>\varepsilon} \max _{0 \leqq t \leqq A(s)-A\left(s^{-}\right)}|q[s](t)-\Phi[\xi(s-), p[s]](t)|\right]=0 .
$$

To do this, we define an $\mathscr{F}_{A(t)}$-point process $q_{n}$ on $\mathscr{W}_{0}^{d}$ as follows:

$$
\begin{aligned}
& \mathbb{D}_{q_{n}}=\mathbb{D} \\
& q_{n}[t](\cdot)=X_{n}((\cdot+A(t-)) \wedge A(t))-X_{n}(A(t-)) \quad t \in \mathbb{D}_{q_{n}} .
\end{aligned}
$$

This $q_{n}$ is well-defined, since

$$
X_{n}^{d}(t)=B^{d}(t)+\int_{0}^{t} \beta^{d}\left(X_{n}^{\prime}\left([s]_{n}\right)\right) d L_{s}^{0}\left(X^{d}\right) .
$$

And it is easily seen that for any $s \in \mathbb{D}$

$$
q_{n}[s](\cdot)=\mathbb{X}_{n}\left(X_{n}(A(s-)), X_{n}\left([A(s-)]_{n}\right), A(s-) ; \cdot, p[s]\right)-X_{n}(A(s-)) .
$$

Hence, from this and the definition of $q$ and $q_{n}$ we observe that the left hand side of (2.17) is dominated by

$$
E\left[\sum_{0<s<L(T)} \chi_{A(s)-A(s-)>\varepsilon} \max _{0 \leqq t \leqq A(s)-A\left(s^{-}\right)} \mid X(t+A(s-))-X_{n}(t+A(s-))\right.
$$




$$
\begin{aligned}
& \left.-\left\{X(A(s-))-X_{n}(A(s-))\right\} \mid\right] \\
& +E\left[\sum_{0<s<L(T)} \chi_{A(s)-A(s-)>\varepsilon}\right. \\
& \quad \times \max _{0 \leqq t \leqq A(s)-A(s-)} \mid \mathbb{X}_{n}\left(X_{n}(A(s-)), X_{n}\left([A(s-)]_{n}\right), A(s-) ; t, p[s]\right) \\
& \left.\quad-X_{n}\left(X_{n}(A(s-)), X_{n}(A(s-)), A(s-) ; t, p[s]\right) \mid\right] \\
& +E\left[\sum_{0<s<L(T)} \chi_{A(s)-A(s-)>\varepsilon}\right. \\
& \quad \times \max _{0 \leqq t \leqq A(s)-A\left(s^{-}\right)} \mid \mathbb{X}_{n}\left(X_{n}(A(s-)), X_{n}(A(s-)), A(s-) ; t, p[s]\right)-X_{n}(A(s-)) \\
& \left.\quad-\left(\mathbb{X}_{1, \xi(s-)}(t, p[s])-(\xi(s-), 0)\right) \mid\right] .
\end{aligned}
$$

Noting that $A(s)<A(L(T)-) \leqq T$ for $0<s<L(T)$ and $\left[X_{n}\left([A(s-)]_{n}\right) ; s \geqq 0\right]$ is $\mathscr{F}_{A(s)}$-predictable, we see that each term of (2.18) is also dominated as follows:

$$
\begin{aligned}
& \text { the first term } \leqq 2 E\left[\max _{0 \leqq t \leqq T}\left|X(t)-X_{n}(t)\right| \int_{0}^{L(T)-} \int_{\mathscr{\mathscr { W }}}^{d} \chi_{\sigma(w)>\varepsilon} N_{p}(d s d w)\right] \text {, } \\
& \text { the second term } \leqq E\left[\int_{0}^{L(T)-} \int_{\mathscr{W}} \chi_{0} \chi_{\sigma(w)>\varepsilon}\right. \\
& \left.\times \max _{0 \leqq t \leqq T}\left|\mathbb{X}_{n}(x, y, \eta ; t, w)-\mathbb{X}_{n}(x, x, \eta ; t, w)\right|_{\substack{x=X_{n}(A(s-)) \\
y=X_{n}\left([A(s-)]_{n}\right) \\
\eta=A(s-)}} N_{p}(d s d w)\right] \\
& =E\left[\int_{0}^{L(T)} \int_{\mathscr{W}}^{d} \chi_{\sigma(w)>\varepsilon}\right. \\
& \left.\times \max _{0 \leqq t \leqq T}\left|X_{n}(x, y, \eta ; t, w)-\mathbb{X}_{n}(x, x, \eta ; t, w)\right|_{\substack{x=X_{n}(A(s-)) \\
y=X_{n}\left([A(s-)]_{n}\right) \\
\eta=A\left(s^{-}\right)}} \hat{N}_{p}(d s d w)\right],
\end{aligned}
$$

the third term $\leqq E\left[\int_{0}^{L(T)-} \int_{\mathscr{V}_{0}^{d}} \chi_{\sigma(w)>\varepsilon}\right.$

$$
\begin{aligned}
& \left.\times \max _{0 \leqq t \leqq T}\left|X_{n}(x, x, \eta ; t, w)-x-\left(X_{1, \xi}(t, w)-(\xi, 0)\right)\right|_{\substack{x=X_{n}(A(s-)) \\
\xi=\xi(s-) \\
\eta=A(s-)}} N_{p}(d s d w)\right] \\
= & E\left[\int_{0}^{L(T)} \int_{\mathscr{V}_{0}^{d}} \chi_{\sigma(w)>\varepsilon}\right. \\
& \left.\times \max _{0 \leqq t \leqq T}\left|\mathbb{X}_{n}(x, x, \eta ; t, w)-x-\left(\mathbb{X}_{1, \xi}(t, w)-(\xi, 0)\right)\right|_{\substack{x=X_{n}(A(s-)) \\
\xi \xi=\xi(s-) \\
\eta=A\left(s^{-}\right)}} \hat{N}_{p}(d s d w)\right] .
\end{aligned}
$$

Therefore, by (1.7), (1.9), (2.16) and Proposition 2.1 it follows that each term of (2.18) tends to zero as $n \uparrow \infty$, which implies (2.17)

图

Thirdly, we state the following, which plays an important role in the proof of Proposition 2.4 below.

Proposition 2.3. Let $f: \mathbb{R}^{d} \rightarrow \mathbb{R}^{1}$ be bounded Lipschitz continuous. Then for each $t>0$ it holds that 


$$
\begin{aligned}
& \underset{\varepsilon \downarrow 0}{\lim . \mathrm{m}} \underbrace{}_{s ; A(s)-A\left(s^{-}\right)>\varepsilon} \int_{A\left(s^{-}\right) \wedge t}^{A(s) \wedge t} f(X(\theta)) d B^{i}(\theta) \\
& \quad=\int_{0}^{t} f(X(\theta)) d B^{i}(\theta)+\delta_{d}^{i} \int_{0}^{L(t)} f(\xi(s-), 0) \beta^{d}(\xi(s-)) d s .
\end{aligned}
$$

Here $L(t)$ stands for $L_{t}^{0}\left(X^{d}\right)$.

For the proof of this proposition, we present the following lemma:

Lemman 2.2. With probability one, it holds that for any $s \in \mathbb{D}$ and $t \geqq 0$

$$
\int_{A(s-) \wedge t}^{A(s) \wedge t} f(X(\theta)) d B^{i}(\theta)=\mathbb{Z}_{f}^{i}\left(1, \xi(s-),(t-A(s-))^{+}, p[s]\right) .
$$

Proof. We take $X_{n}$ introduced just before Lemma 2.1 and set

$$
I^{i}(t)=\int_{0}^{t} f(X(\theta)) d B^{i}(\theta), \quad I_{n}^{i}(t)=\int_{0}^{t} f\left(X_{n}\left([\theta]_{n}\right)\right) d B^{i}(\theta) .
$$

Then we easily see by $\mathbb{L e m m a} 2.1$ that $\lim _{n \uparrow \infty} \mathbb{E}\left[\max _{0 \leqq s \leqq T}\left|I^{i}(s)-I_{n}^{i}(s)\right|^{2}\right]=0$ for each $T>0$, and so it follows that

$$
\begin{aligned}
\lim _{n \uparrow \infty} E\left[\max _{0 \leqq t \leqq T} \sum_{s ; A(s)-A(s-)>\varepsilon} \mid I^{i}(A(s) \wedge t)-I^{i}(A(s-) \wedge t)\right. \\
\left.-\left(I_{n}^{i}(A(s) \wedge t)-I_{n}^{i}(A(s-) \wedge t)\right) \mid\right]=0
\end{aligned}
$$

for each $\varepsilon>0$ and $T>0$. We next define $\mathbb{I}_{n}^{i}\left(t, S, S^{\prime}\right)\left(t \geqq 0, S^{\prime} \geqq S \geqq 0\right)$ by

$$
\begin{aligned}
\mathbb{I}_{n}^{i}\left(t, S, S^{\prime}\right) & \\
= & \sum_{k=1}^{\infty} f\left(X_{n}\left(\left(S+\left(\frac{k-1}{2^{n}}-S\right)^{+} \wedge(t-S)^{+}\right) \wedge S^{\prime}\right)\right) \\
& \times\left(B^{i}\left(\left(S+\left(\frac{k}{2^{u}}-S\right)^{+} \wedge(t-S)^{+}\right) \wedge S^{\prime}\right)-B^{i}\left(\left(S+\left(\frac{k-1}{2^{n}}-S\right)^{+}\right.\right.\right. \\
& \left.\left.\left.\wedge(t-S)^{+}\right) \wedge S^{\prime}\right)\right) .
\end{aligned}
$$

Then, since $\left|I_{n}^{i}\left(t, S, S^{\prime}\right)-\left(I_{n}^{i}\left(t \wedge S^{\prime}\right)-I_{n}^{i}(t \wedge S)\right)\right|$ is dominated by $2 \max _{0 \leqq s \leqq t}\left|B^{i}(s)\right|$ $\left|f\left(X_{n}(t \wedge S)\right)-f\left(X_{n}\left([t \wedge S]_{n}\right)\right)\right|$, we observe from (2.15) that

$$
\lim _{n \uparrow \infty} \mathbb{E}\left[\max _{\substack{0 \leq t \leq T \\ S^{\prime} \geqq S \geqq 0}}\left|I_{n}^{i}\left(t \wedge S^{\prime}\right)-I_{n}^{i}(t \wedge S)-I_{n}^{i}\left(t, S, S^{\prime}\right)\right|^{2}\right]=0
$$

for each $T>0$. Hence it follows that

(2.20) $\lim _{n \uparrow \infty} \mathbb{E}\left[\max _{0 \leqq t \leqq T} \sum_{s ; A(s)-A(s-)>\varepsilon} \mid I_{n}^{i}(A(s) \wedge t)-I_{n}^{i}(A(s-) \wedge t)\right.$

$$
\left.-\mathbb{I}_{n}^{i}(t, A(s-), A(s)) \mid\right]=0
$$

for each $\varepsilon>0$ and $T>0$. On the other hand, since it can be easily verified that for any $s \in \mathbb{D}$ 


$$
\begin{aligned}
& I_{n}^{i}(t, A(s-), A(s)) \\
& =\mathbb{I}_{n}^{i}\left(X_{n}(A(s-)), X_{n}\left([A(s-)]_{n}\right), A(s-) ;(t-A(s-))^{+}, p[s]\right),
\end{aligned}
$$

from (2.16), Corollary 1.1 and Proposition 2.1 we observe that

$$
\begin{aligned}
& \lim _{n \uparrow \infty} E\left[\max _{0 \leqq t \leqq T} \sum_{s ; A(s)-A\left(s^{-}\right)>\varepsilon} \mid I_{n}^{i}(t, A(s-), A(s))-I_{f}^{i}(1, \xi(s-),\right. \\
& \left.\left.(t-A(s-))^{+}, p[s]\right) \mid\right] \\
& \leqq \\
& \quad \lim _{n \uparrow \infty} E\left[\int_{0}^{L(T)} \int_{\mathscr{F}_{0}^{d}} \chi_{\sigma(w)>\varepsilon}\right. \\
& \left.\quad \times \max _{0 \leqq u \leqq T}\left|I_{n}^{i}(x, y, \eta ; u, w)-I_{f}^{i}(1, \xi, u, w)\right|_{\substack{x=X_{n}(A(s-)) \\
y=X_{n}\left([A(s)]_{n}\right) \\
\xi=\xi(s-), \eta=A(s-)}} \hat{N}_{p}(d s d w)\right] \\
& =0
\end{aligned}
$$

for each $\varepsilon>0$ and $T>0$. Thus, by putting (2.19), (2.20) and (2.21) together, the lemma follows immediately.

To prove Proposition 2.3, we must trace the proof of Theorem 2 in [8] almost similarly. We shall here sketch the proof.

A sketch of the proof of Proposition 2.3. For simplicity we denote $\mathscr{F}_{A(t)}$ by $\widetilde{\mathscr{F}}_{t^{\circ}}$ Setting $p[t]=q[t]=0$ if $t \notin D$, we define a family $\left(\mathscr{H}_{t}\right)_{t>0}$ of sub $\sigma$-fields of $\mathscr{F}$ by

$$
\mathscr{H}_{t}=\widetilde{\mathscr{F}}_{L(t)-} \vee \sigma\left(p[L(t)]_{t-A(L(t)-)}^{\cdot}, q[L(t)]_{t-A(L(t)-)}^{\cdot}\right) .
$$

Then it is easily checked that $\mathscr{H}_{t} \subset \mathscr{H}_{t^{\prime}}$ for $0<t<t^{\prime}$ and

$$
\sigma(X(s), B(s) ; 0 \leqq s \leqq t) \subset \mathscr{H}_{t} \subset \mathscr{F}_{t} \subset \widetilde{\mathscr{F}}_{L(t)} \quad t>0 .
$$

For a bounded Lipschitz continuous $f$, we observe by Lemma 2.2 that for each $t>0$ and $\varepsilon>0$

$$
\begin{aligned}
& \sum_{s ; A(s)-A\left(s^{-}\right)>\varepsilon} \int_{A\left(s^{-}\right) \wedge t}^{A(s) \wedge t} f(X(\theta)) d B^{i}(\theta) \\
= & \int_{0}^{L(t)+} \int_{\mathscr{W} d}^{d} \chi_{\sigma(w)>\varepsilon} I_{f}^{i}\left(1, \xi(s-),(t-A(s-))^{+}, w\right) \tilde{N}_{p}(d s d w) \\
& +\int_{0}^{L(t)} \int_{\mathscr{F}_{0}^{d}} \chi_{\sigma(w)>\varepsilon} I_{f}^{i}\left(1, \xi(s-),(t-A(s-))^{+}, w\right) \hat{N}_{p}(d s d w) .
\end{aligned}
$$

We denote by $M_{f, \varepsilon}^{i}(t)$ and $V_{f, \varepsilon}^{i}(t)$, the first term and second term in the right hand side, respectively. Also we define $M_{f}^{i}(t)$ and $V_{f}^{i}(t)$ as follows:

$$
\begin{aligned}
& M_{f}^{i}(t)=\int_{0}^{L(t)+} \int_{\mathscr{P}_{0}^{d}} \mathbb{I}_{f}^{i}\left(1, \xi(s-),(t-A(s-))^{+}, w\right) \tilde{N}_{p}(d s d w) \\
& V_{f}^{i}(t)=\delta_{d}^{i} \int_{0}^{L(t)} f(\xi(s-), 0) \beta^{d}(\xi(s-)) d s .
\end{aligned}
$$


Then we see that for each $t>0$

$$
\underset{\varepsilon \downarrow 0}{\text { 1.i.m. }} M_{f, \varepsilon}^{i}(t)=M_{f}^{i}(t), \underset{\varepsilon \downarrow 0}{\operatorname{li} \text { i.m. }} V_{f, \varepsilon}^{i}(t)=V_{f}^{i}(t),
$$

Hence we have that for each $t>0$

$$
\underset{\varepsilon \downarrow 0}{\text { 1.i.m. }} \sum_{s ; A(s)-A\left(s^{-}\right)>\varepsilon} \int_{A\left(s^{-}\right) \wedge t}^{A(s) \wedge t} f(X(\theta)) d B^{i}(\theta)=M_{f}^{i}(t)+V_{f}^{i}(t) .
$$

Therefore, if we show that for each $t>0$

$$
M_{f}^{i}(t)=\int_{0}^{t} f(X(\theta)) d B^{i}(\theta) \text { a.s. }(P),
$$

then the proof of Proposition 2.3 is completed. To do this, let $t^{\prime}>t>0$, let $\left[G_{1}(u) ; u \geqq 0\right]$ be a real bounded $\widetilde{\mathscr{F}}_{u}$-predictable process and let $G_{2}: \mathscr{W}^{2 d} \rightarrow \mathbb{R}^{1}$ be bounded Borel measurable. Setting a bounded $\mathscr{H}_{t}$-measurable $H$ by

$$
H=G_{1}(L(t)) G_{2}\left(p[L(t)]_{t-A(L(t)-)}^{\cdot}, q[L(t)]_{t-A(L(t)-)}^{\cdot}\right),
$$

by the same way as in the proof of Theorem 2 in [8] we can show that

$$
\begin{aligned}
& \mathbb{E}\left[\left(M_{f, \varepsilon}^{i}\left(t^{\prime}\right)-M_{f, \varepsilon}^{i}(t)\right) H\right]=o(1) \text { as } \varepsilon \downarrow 0 \\
& \mathbb{E}\left[\left(M_{f, \varepsilon}^{i}\left(t^{\prime}\right)^{2}-\int_{0}^{t^{\prime}} f(X(\theta))^{2} d \theta-\left\{M_{f, \varepsilon}^{i}(t)^{2}-\int_{0}^{t} f(X(\theta))^{2} d \theta\right\}\right) H\right]=o(1)
\end{aligned}
$$
as $\varepsilon \downarrow 0$.

Hence, by (2.23) we obtain that

$$
\begin{aligned}
& E\left[M_{f}^{i}\left(t^{\prime}\right) H\right]=E\left[M_{f}^{i}(t) H\right] \\
& E\left[\left(M_{f}^{i}\left(t^{\prime}\right)^{2}-\int_{0}^{t^{\prime}} f(X(\theta))^{2} d \theta\right) H\right]=E\left[\left(M_{f}^{i}(t)^{2}-\int_{0}^{t} f(X(\theta))^{2} d \theta\right) H\right] .
\end{aligned}
$$

Thus, noting that $M_{f}^{i}(u)$ and $\int_{0}^{u} f(X(\theta))^{2} d \theta$ are $\mathscr{H}_{u}$-measurable a.s. $(P)$ for each $u>0$, from the arbitrariness of $t^{\prime}>t>0, G_{1}$ and $G_{2}$ we see that for a bounded Lipschitz continuous $f$

$$
\left[M_{f}^{i}(t) ; t>0\right] \text { and }\left[M_{f}^{i}(t)^{2}-\int_{0}^{t} f(X(\theta))^{2} d \theta ; t>0\right] \text { are } \mathscr{H}_{t} \text {-martingales. }
$$

Further, from the linearity of $\mathbb{I}_{f}^{i}$ with respect to $f$ and (2.25), we see that for a bounded Lipschitz continuous $f$ and $g$

$$
\left[M_{f}^{i}(t) M_{g}^{i}(t)-\int_{0}^{t} f(X(\theta)) g(X(\theta)) d \theta ; t>0\right] \text { is also an } \mathscr{H}_{t} \text {-martingale. }
$$

On the other hand, it can be seen that $M_{1}^{i}(t)=B^{i}(t)$ a.s. $(P)$ for each $t>0$. Therefore, by this, (2.22), (2.25) and (2.26) we observe that for each $t>0$ 


$$
\begin{aligned}
& E\left[M_{f}^{i}(t) \int_{0}^{t} f(X(\theta)) d B^{i}(\theta)\right] \\
& =\lim _{n \uparrow \infty}\left(\sum_{k=1}^{[t]_{n}} E\left[M_{f}^{i}(t) f\left(X\left((k-1) / 2^{n}\right)\right)\left\{B^{i}\left(k / 2^{n}\right)-B^{i}\left((k-1) / 2^{n}\right)\right\}\right]\right. \\
& \left.\quad+E\left[M_{f}^{i}(t) f\left(X\left([t]_{n}\right)\right)\left\{B^{i}(t)-B^{i}\left([t]_{n}\right)\right\}\right]\right) \\
& =\lim _{n \uparrow \infty}\left(E\left[M_{f}^{i}\left(1 / 2^{n}\right) f(X(0))\left\{B^{i}\left(1 / 2^{n}\right)-B^{i}(0)\right\}\right]\right. \\
& \left.\quad+E\left[\int_{0}^{t} f\left(X\left([\theta]_{n}\right)\right) f(X(\theta)) d \theta\right]-E\left[\int_{0}^{1 / 2^{n}} f(X(\theta)) d \theta f(X(0))\right]\right) \\
& =E\left[\int_{0}^{t} f(X(\theta))^{2} d \theta\right],
\end{aligned}
$$

so that it follows that $E\left[\left(M_{f}^{i}(t)-\int_{0}^{t} f(X(\theta)) d B^{i}(\theta)\right)^{2}\right]=0$ for each $t>0$. Consequently we have (2.24), and complete the proof of the proposition. 圈

Fourthly, using Proposition 1.4 and 2.3, we shall derive an SDE of jump type which $\xi(t)$ satisfies: We define an $\mathscr{F}_{A(t)}-B M_{0}^{d} B^{*}(t)$ and a bounded Lipschitz continuous $\tilde{\beta}: \mathbb{R}^{d-1} \rightarrow \mathbb{R}^{d-1}$ as follows:

$$
\begin{aligned}
B^{*}(t) & =M(A(t)), \\
\tilde{\beta}^{i}(\xi) & =\beta^{i}(\xi) \\
& +c^{+}(\xi)^{2} E^{Q^{d,+}}\left[\int_{0}^{\sigma(w)} b^{i}\left(X_{c^{+}(\xi), \xi}(s)\right) d s ; \sigma(w) \leqq 1\right] \\
& +c^{-}(\xi)^{2} E^{Q^{d,-}}\left[\int_{0}^{\sigma(w)} b^{i}\left(\mathbb{X}_{c^{-}(\xi), \xi}(s)\right) d s ; \sigma(w) \leqq 1\right] \\
& -c^{+}(\xi) E^{Q^{d,+}}\left[\int_{0}^{1} a_{d}^{i}\left(\mathbb{X}_{c^{+}(\xi), \xi}(s)\right) d w^{d}(s) ; \sigma(w)>1\right] \\
& -c^{-}(\xi) E^{Q^{d,-}}\left[\int_{0}^{1} a_{d}^{i}\left(\mathbb{X}_{c^{-}(\xi), \xi}(s)\right) d w^{d}(s) ; \sigma(w)>1\right] \quad i=1, \ldots, d-1 .
\end{aligned}
$$

And we define $c(\xi, w)\left(\xi \in \mathbb{R}^{d-1}, w \in \mathscr{W}_{0}^{d}\right)$ by

$$
c(\xi, w)=c^{+}(\xi) \chi_{\mathscr{W}}^{d,+}(w)+c^{-}(\xi) \chi_{\mathscr{W}}^{d,-}(w) .
$$

Then we have

Proposition 2.4. An $\mathscr{F}_{A(t)}$-adapted $(d-1)$-dimensional right continuous process $\xi(t)$ satisfies the following SDE of jump type:

$$
\begin{aligned}
& \xi^{i}(t)=\xi_{0}^{i}+\sum_{j=1}^{d} \int_{0}^{t} \tau_{j}^{i}(\xi(\theta)) d B^{* j}(\theta)+\int_{0}^{t} \tilde{\beta}^{i}(\xi(\theta)) d \theta \\
& +\int_{0}^{t+} \int_{\mathscr{V}_{0}^{d}} \chi_{c(\xi(s-), w)>0} \chi_{\sigma(w)>c(\xi(s-), w)^{2}} \Phi[\xi(s-), w]^{i}(\sigma(w)) N_{p}(d s d w) \\
& +\int_{0}^{t+} \int_{\mathscr{r}_{0}^{d}} \chi_{c(\xi(s-), w)>0} \chi_{\sigma(w) \leqq c(\xi(s-), w)^{2}} \Phi[\xi(s-), w]^{i}(\sigma(w)) \tilde{N}_{p}(d s d w) \\
& i=1, \ldots, d-1 .
\end{aligned}
$$


Proof. First of all we note the following: With probability one, it holds that $c(\xi(s-), p[s])>0$ for any $s \in \mathbb{D}$ and

$$
\#\{s \in \mathbb{D} ; 0<s \leqq t, A(s)-A(s-)>\varepsilon c(\xi(s-), p[s])\}<+\infty \quad \text { for any } t>0 \text {. }
$$

We define $\mathscr{F}_{A(t)}$-adapted $(d-1)$-dimensional right continuous processes $\zeta_{\varepsilon}(t)$, $\bar{\zeta}_{\varepsilon}(t)$ and $\zeta(t)$ as follows:

$$
\begin{aligned}
\zeta_{\varepsilon}(t) & =\sum_{\substack{0<s \leq t \\
s \in \mathbb{D}}} \chi_{A(s)-A(s-)>\varepsilon c(\xi(s-), p[s])^{2}}(\xi(s)-\xi(s-)), \\
\bar{\zeta}_{\varepsilon}(t) & =\sum_{\substack{0<s \leq t \\
s \in \mathbb{D}}} \chi_{A(s)-A(s-)>\varepsilon}(\xi(s)-\xi(s-)), \\
\zeta(t) & =\left.\int_{0}^{t}\left\{\tilde{\beta}(\xi)-\beta^{\prime}(\xi)+\beta^{d}(\xi) a_{d}^{\prime}(\xi, 0)\right\}\right|_{\xi=\xi(s)} d s \\
& +\int_{0}^{t+} \int_{\mathscr{W}_{0}^{d}} \chi_{c(\xi(s-), w)>0} \chi_{\sigma(w)>c(\xi(s-), w)^{2}} \Phi[\xi(s-), w]^{\prime}(\sigma(w)) N_{p}(d s d w) \\
& +\int_{0}^{t+} \int_{\mathscr{F}_{0}^{d}} \chi_{c(\xi(s-), w)>0} \chi_{\sigma(w) \leqq c(\xi(s-), w)^{2}} \Phi[\xi(s-), w]^{\prime}(\sigma(w)) \tilde{N}_{p}(d s d w) .
\end{aligned}
$$

From the above note and the estimate $(1.15), \zeta_{\varepsilon}(t)$ and $\zeta(t)$ are well-defined. We first show that for each $T>0$

$$
\lim _{\varepsilon \downarrow 0} E\left[\sup _{0 \leqq t \leqq T}\left|\zeta_{\varepsilon}(t)-\zeta(t)\right|^{2}\right]=0 .
$$

From the definition of the point process $q$ and Proposition 2.2, we observe that

$$
\begin{aligned}
\zeta_{\varepsilon}(t) & =\sum_{0<s \leqq t} \chi_{\sigma(p[s])>\varepsilon c(\xi(s-), p[s])^{2}} \Phi[\xi(s-), p[s]]^{\prime}(\sigma(p[s])) \\
& =\int_{0}^{t+} \int_{\mathscr{W}_{0}^{d}} \chi_{c(\xi(s-), w)>0} \chi_{\sigma(w)>\varepsilon c(\xi(s-), w)^{2}} \Phi[\xi(s-), w]^{\prime}(\sigma(w)) N_{p}(d s d w) .
\end{aligned}
$$

By virtue of the estimates (1.15) and (1.16), the last hand side is written as

$$
\begin{gathered}
\int_{0}^{t+} \int_{\mathscr{F}_{0}^{d}} \chi_{c(\xi(s-), w)>0} \chi_{\sigma(w)>c(\xi(s-), w)^{2}} \Phi[\xi(s-), w]^{\prime}(\sigma(w)) N_{p}(d s d w) \\
+\int_{0}^{t+} \int_{\mathscr{P}_{0}^{d}} \chi_{c(\xi(s-), w)>0} \chi_{\sigma(w) \leqq c(\xi(s-), w)^{2}} \Phi[\xi(s-), w]^{\prime}(\sigma(w)) \tilde{N}_{p}(d s d w) \\
-\int_{0}^{t+} \int_{\mathscr{V}_{0}^{d}} \chi_{c(\xi(s-), w)>0} \chi_{\sigma(w) \leqq \varepsilon c(\xi(s-), w)^{2}} \Phi[\xi(s-), w]^{\prime}(\sigma(w)) \tilde{N}_{p}(d s d w) \\
+\int_{0}^{t+} \int_{\mathscr{F}_{0}^{d}} \chi_{c(\xi(s-), w)>0} \chi_{\varepsilon c(\xi(s-), w)^{2}<\sigma(w) \leqq c(\xi(s-), w)^{2}} \\
\times \Phi[\xi(s-), w]^{\prime}(\sigma(w)) \hat{N}_{p}(d s d w),
\end{gathered}
$$

in which $0<\varepsilon<1$. For the third and the fourth term in this expression, the following is true: $\mathrm{By}(1.15)$,

$$
\lim _{\varepsilon \downarrow 0} \mathbb{E}\left[\sup _{0 \leqq t \leqq T} \mid \text { the third term }\left.\right|^{2}\right]=0 .
$$


By (1.17) and by noting that $c^{+}(\xi)-c^{-}(\xi)=\beta^{d}(\xi)$,

$\lim _{\varepsilon \downarrow 0} \sup _{0 \leqq t \leqq T} \mid$ the fourth term $-\left.\int_{0}^{t}\left\{\tilde{\beta}(\xi)-\beta^{\prime}(\xi)+\beta^{d}(\xi) a_{d}^{\prime}(\xi, 0)\right\}\right|_{\xi=\xi(s)} d s \mid=0$.

Hence, putting all this together, we immediately obtain (2.27). Applying (1.18) instead of (1.17), by the same way as above we see that for each $T>0$

$$
\lim _{\varepsilon \downarrow 0} E\left[\sup _{0 \leqq t \leqq T}\left|\zeta_{\varepsilon}(t)-\bar{\zeta}_{\varepsilon}(t)\right|^{2}\right]=0 .
$$

Next, from (2.7) the following is obvious: For each $s>0$ and $t \geqq 0$

$$
\begin{aligned}
& X^{\prime}(A(s) \wedge t)-X^{\prime}(A(s-) \wedge t) \\
& =\sum_{j=1}^{d} \int_{A\left(s^{-}\right) \wedge t}^{A(s) \wedge t} a_{j}^{\prime}(X(\theta)) d B^{j}(\theta)+\int_{A\left(s^{-}\right) \wedge t}^{A(s) \wedge t} b^{\prime}(X(\theta)) d \theta \\
& \quad+\sum_{j=1}^{d} \int_{A\left(s^{-}\right) \wedge t}^{A(s) \wedge t} \tau_{j}^{\prime}(\Xi(\theta)) d M^{j}(\theta)+\int_{A\left(s^{-}\right) \wedge t}^{A(s) \wedge t} \beta^{\prime}(\Xi(\theta)) d L(\theta)
\end{aligned}
$$

where $L(\theta)=L_{\theta}^{0}\left(X^{d}\right)$. But, from the fact that $\int_{A\left(s^{-}\right) \wedge t}^{A(s) \wedge t} d L(\theta)=0$ for $s>0$ and $t \geqq 0$, the last two terms in the above formula are identically zero. Hence, for each $t>0$, summing up over $0<s \leqq L(t)$ such that $A(s)-A(s-)>\varepsilon$, we have

$$
\begin{gathered}
\bar{\zeta}_{\varepsilon}(L(t)-)+\chi_{A(L(t))-A(L(t)-)>\varepsilon}\left(X^{\prime}(t)-\xi(L(t)-)\right) \\
=\sum_{j=1}^{d} \sum_{s ; A(s)-A\left(s^{-}\right)>\varepsilon} \int_{A\left(s^{-}\right) \wedge t}^{A(s) \wedge t} a_{j}^{\prime}(X(\theta)) d B^{j}(\theta) \\
\quad+\sum_{s ; A(s)-A\left(s^{-}\right)>\varepsilon} \int_{A\left(s^{-}\right) \wedge t}^{A(s) \wedge t} b^{\prime}(X(\theta)) d \theta .
\end{gathered}
$$

Here, by applying Proposition 2.3, the first term in the right hand side of (2.29) converges in $L^{2}$, as $\varepsilon \downarrow 0$, to

$$
\sum_{j=1}^{d} \int_{0}^{t} a_{j}^{\prime}(X(\theta)) d B^{j}(\theta)+\int_{0}^{L(t)} a_{d}^{\prime}(\xi(\theta), 0) \beta^{d}(\xi(\theta)) d \theta .
$$

Also, it is clear that the second term converges, as $\varepsilon \downarrow 0$, to $\int_{0}^{t} b^{\prime}(X(\theta)) d \theta$ a.s. $(P)$, because the Lebesgue measure of the set $(0, \infty) \backslash \cup_{s \in \boldsymbol{D}}(A(s-), A(s))$ is zero a.s. $(P)$. Therefore, we conclude that the right hand side of (2.29) converges in $L^{2}$, as $\varepsilon \downarrow 0$, to

$$
\sum_{j=1}^{d} \int_{0}^{t} a_{j}^{\prime}(X(\theta)) d B^{j}(\theta)+\int_{0}^{t} b^{\prime}(X(\theta)) d \theta+\int_{0}^{L(t)} a_{d}^{\prime}(\xi(\theta), 0) \beta^{d}(\xi(\theta)) d \theta
$$

that is,

$$
\begin{aligned}
& X^{\prime}(t)-\xi_{0}-\sum_{j=1}^{d} \int_{0}^{t} \tau_{j}^{\prime}(\Xi(\theta)) d M^{j}(\theta)-\int_{0}^{L(t)} \beta^{\prime}(\xi(\theta)) d \theta \\
& \quad+\int_{0}^{L(t)} a_{d}^{\prime}(\xi(\theta), 0) \beta^{d}(\xi(\theta)) d \theta
\end{aligned}
$$


where we have to recall (2.7). On the other hand, by (2.27) and (2.28), the left hand side of (2.29) converges in probability to $\zeta(L(t)-)+X^{\prime}(t)-\xi(L(t)-)$ as $\varepsilon \downarrow 0$. Thus, combining with these, we have that for each $t>0$

$$
\begin{aligned}
& \xi(L(t)-)=\xi_{0}+\sum_{j=1}^{d} \int_{0}^{t} \tau_{j}^{\prime}(\Xi(\theta)) d M^{j}(\theta)+\int_{0}^{L(t)} \tilde{\beta}(\xi(\theta)) d \theta \\
& \quad+\int_{0}^{L(t)-} \int_{\mathscr{V}_{0}^{d}} \chi_{c(\xi(s-), w)>0} \chi_{\sigma(w)>c(\xi(s-), w)^{2}} \Phi[\xi(s-), w]^{\prime}(\sigma(w)) N_{p}(d s d w) \\
& \quad+\int_{0}^{L(t)-} \int_{\mathscr{O}_{0}^{d}} \chi_{c(\xi(s-), w)>0} \chi_{\sigma(w) \leqq c(\xi(s-), w)^{2}} \Phi[\xi(s-), w]^{\prime}(\sigma(w)) \tilde{N}_{p}(d s d w)
\end{aligned}
$$

a.s. $(P)$.

But, since both sides are left continuous in $t$, we see that this identity holds for all $t>0$ a.s. $(P)$. Consequently, replacing $t$ by $A(t)$ and then taking the right hand limits in $t$, we obtain the conclusion of the proposition.

Putting the above propositions together, we shall prove (2.6).

Proof of (2.6). As before, we denote $\mathscr{F}_{A(t)}$ by $\widetilde{\mathscr{F}}_{t}$. We now take a filtered probability space $\left(\Omega^{\prime}, \mathscr{F}^{\prime}, P^{\prime}, \mathscr{F}_{t}^{\prime}\right)$ and an $\mathscr{F}_{t}^{\prime}$-stationary Poisson point process $p^{\prime}$ on $\mathscr{W}_{0}^{d}$ with characteristic measure $Q^{d}(d w)$. And we put a filtered probability space $\left(\bar{\Omega}, \overline{\mathscr{F}}, \bar{P}, \overline{\mathscr{F}}_{t}\right)$ as follows:

$$
\bar{\Omega}=\Omega \times \Omega^{\prime}, \quad \overline{\mathscr{F}}=\mathscr{F} \times \mathscr{F}^{\prime}, \quad \bar{P}=P \times P^{\prime}, \quad \overline{\mathscr{F}}_{t}=\bigcap_{\varepsilon>0}\left(\widetilde{\mathscr{F}}_{t+\varepsilon} \times \mathscr{F}_{t+\varepsilon}^{\prime}\right) .
$$

Then we may regard the processes $\xi(\cdot), B^{*}(\cdot), p, p^{\prime} \cdots$ as defined on $\left(\bar{\Omega}, \overline{\mathscr{F}}, \bar{P}, \overline{\mathscr{F}}_{t}\right)$. Clearly $B^{*}$ is an $\overline{\mathscr{F}}_{t}-B M_{0}^{d}$. Noting that $c(\xi(s-), p[s])>0$ for any $s \in \mathbb{D}_{p}$ and $\mathbb{D}_{p} \cap \mathbb{D}_{p^{\prime}}=\phi$ a.s. $(\bar{P})$, we define an $\overline{\mathscr{F}}_{t}$-point process $\bar{p}$ on $\mathscr{W}_{0}^{d}$ as follows:

$$
\begin{aligned}
& \mathbb{D}_{\bar{p}}=\mathbb{D}_{p} \cup\left\{s \in \mathbb{D}_{p^{\prime}} ; c\left(\xi(s-), p^{\prime}[s]\right)=0\right\} \\
& \bar{p}[s]= \begin{cases}T_{c(\xi(s-), p[s])^{-1}} p[s] & \text { for } s \in \mathbb{D}_{p} \\
p^{\prime}[s] & \text { for } s \in \mathbb{D}_{p^{\prime}} \quad \text { such as } c\left(\xi(s-), p^{\prime}[s]\right)=0 .\end{cases}
\end{aligned}
$$

From its definition, it is clear that

$$
\left\{\begin{array}{l}
\mathbb{D}_{p}=\left\{s \in \mathbb{D}_{\bar{p}} ; c(\xi(s-), \bar{p}[s])>0\right\} \\
p[s]=T_{c(\xi(s-), \bar{p}[s])} \bar{p}[s] \quad s \in \mathbb{D}_{p} .
\end{array}\right.
$$

Further, by Proposition 2.1, the point process $\bar{p}$ is an $\overline{\mathscr{F}}_{t}$-stationary Poisson point process on $\mathscr{W}_{0}^{d}$ with characteristic measure $Q^{d}(d w)$.

By (2.30) and Proposition 2.4, the $\operatorname{SDE}$ which $\xi(t)$ satisfies becomes as follows : 


$$
\begin{aligned}
\xi^{i}(t) & =\xi_{0}^{i}+\sum_{j=1}^{d} \int_{0}^{t} \tau_{j}^{i}(\xi(s)) d B^{* j}(s)+\int_{0}^{t} \tilde{\beta}^{i}(\xi(s)) d s \\
+ & \int_{0}^{t+} \int_{\mathscr{V} d}^{d} \chi_{\sigma(w)>1} \Phi\left[\xi(s-), T_{c(\xi(s-), w)} w\right]^{i}\left(c(\xi(s-), w)^{2} \sigma(w)\right) N_{\bar{p}}(d s d w) \\
+ & \int_{0}^{t+} \int_{\mathscr{\mathscr { V }} d}^{d} \chi_{\sigma(w) \leqq 1} \Phi\left[\xi(s-), T_{c(\xi(s-), w)} w\right]^{i}\left(c(\xi(s-), w)^{2} \sigma(w)\right) \tilde{N}_{\bar{p}}(d s d w) \\
& i=1, \ldots, d-1 .
\end{aligned}
$$

Hence, recalling (1.13), we see that $\xi(t)$ is a solution of the following SDE of jump type:

$$
\begin{aligned}
& \xi^{i}(t)=\xi_{0}^{i}+\sum_{j=1}^{d} \int_{0}^{t} \tau_{j}^{i}(\xi(s)) d B^{* j}(s)+\int_{0}^{t} \tilde{\beta}^{i}(\xi(s)) d s \\
& \quad+\int_{0}^{t+} \int_{\mathscr{N}}^{d} \chi_{\sigma(w)>1} f^{i}(\xi(s-), w) N_{\bar{p}}(d s d w) \\
& \quad+\int_{0}^{t+} \int_{\mathscr{W}_{0}^{d}} \chi_{\sigma(w) \leqq 1} f^{i}(\xi(s-), w) \tilde{N}_{\bar{p}}(d s d w) \quad i=1, \ldots, d-1
\end{aligned}
$$

where $f(\xi, w)=\Xi_{c(\xi, w), \xi}(\sigma(w), w)-\xi$. Therefore, since $\tau$ and $\tilde{\beta}$ are bounded Lipschitz continuous, and since, by (1.5) and (1.14), it holds that

$$
\begin{aligned}
& \sup _{\xi \in \mathbb{R}^{d-1}} E^{Q^{d}}\left[|f(\xi, w)|^{2} ; \sigma(w) \leqq 1\right]<+\infty \\
& E^{Q^{d}}\left[\left|f(\xi, w)-f\left(\xi^{\prime}, w\right)\right|^{2} ; \sigma(w) \leqq 1\right] \leqq \text { const. }\left|\xi-\xi^{\prime}\right|^{2} \quad \xi, \xi^{\prime} \in \mathbb{R}^{d-1},
\end{aligned}
$$

we apply Theorem IV-9.1 in [2] to obtain the following:

(2.31) An $\overline{\mathscr{F}}_{t}$-adapted (d-1)-dimensional right continuous process $\xi(t)$ is a functional, which is uniquely determined from $\tau, \tilde{\beta}$ and $f$, of $\xi_{0}$, an

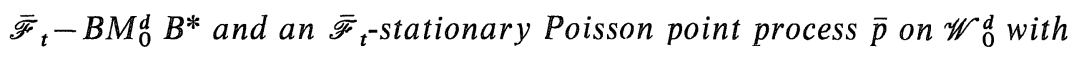
characteristic measure $Q^{d}(d w)$.

Also, the following is easily verified:

$$
\left\{\begin{array}{l}
A(t)=\int_{0}^{t+} \int_{\mathscr{r}}^{d d} c(\xi(s-), w)^{2} \sigma(w) N_{\bar{p}}(d s d w) \\
L_{t}^{0}\left(X^{d}\right)=L(t)=\inf \{s ; A(s)>t\} .
\end{array}\right.
$$

And, by Proposition 2.2 and (2.30) it is easy to see that for each $t>0$

$$
\begin{aligned}
& X(t) \\
& =\left\{\begin{array}{c}
\Phi\left[\xi(L(t)-), T_{c(\xi(L(t)-), \bar{p}[L(t)])} \bar{p}[L(t)]\right](t-A(L(t)-))+(\xi(L(t)-), 0) \\
\text { if } L(t) \in \mathbb{D}_{\bar{p}} \text { and } c(\xi(L(t)-), \bar{p}[L(t)])>0 \\
(\xi(L(t)-), 0) \text { otherwise. }
\end{array}\right.
\end{aligned}
$$

Thus, from $(2.31) \sim(2.33)$ we can clarify the structure of $X(\cdot)$, and as its consequence we establish the uniqueness of $\mathbb{S}_{\delta_{\left(\xi_{0}, 0\right)}}\left[a, b, \tau, \beta, \delta_{0}\right]$. 國 


\section{§3. Existernce}

Let $a, b, \tau, \beta$ and $\mu$ satisfy $(0.2)^{\prime},(0.3),(0.4)$ and $(0.5)$, and $\lambda(d x)$ be a probability measure on $\mathbb{R}^{d}$. In this section, we show the nonemptiness of $\mathbb{S}_{\lambda}[a, b, \tau, \beta, \mu]$. The general case is reduced to this case, ifwe use themethods in [7] or Theorem IV-7.2 in [2], i.e., the following methods; transformation of Brownian motion by an orthogonal matrix, time change and transformation of drift.

First we start with the following proposition: Let $\alpha: \mathbb{R}^{1} \rightarrow \mathbb{R}^{1}$ be a bounded and uniformly positive Borel measurable function and let $B(t)$ be an $\mathscr{F}_{t}-B M_{0}^{1}$ defined on a filtered probability space $\left(\Omega, \mathscr{F}, P, \mathscr{F}_{t}\right)$. I et $\gamma:[0, \infty) \times \Omega \times \mathbb{R}^{1}$ $\rightarrow \mathbb{R}^{1}$ be $\mathscr{F}_{t}$-progressively measurable and satisfy $\sup _{s, \omega, \eta}|\gamma(s, \omega, \eta)| \leqq 1$. Suppose we are given a 1 -dimensional continuous $\mathscr{F}_{t}$-semimartingale $Y(t)$ satisfying

$$
Y(t)=Y(0)+\int_{0}^{t} \alpha(Y(s)) d B(s)+\int_{0}^{t} \gamma(s, Y(s)) d \mathbb{L}_{s}^{\delta} \mathbb{Z}(Y)
$$

where $\mathbb{L}_{t}^{\delta} Z(Y)=\sum_{k \in Z} L_{t}^{k}(Y)$. Then we have

Proposition 3.11. For any $p>0$ there exists a constant $C$ depending only on $p$ and $\sup _{\eta \in \mathbb{R}^{1}} \alpha(\eta)$ such that

$$
\mathbb{E}\left[\left|\mathbb{L}_{t}^{\delta} Z(Y)-L_{t}^{\delta} Z(Y)\right|^{2 p}\right] \leqq C\left(1+\left|t^{\prime}-t\right|^{p}\right)\left|t^{\prime}-t\right|^{p} \quad \text { for } \quad t, t^{\prime} \geqq 0 .
$$

Proof. By time change by means of $\int_{0}^{t} \alpha(Y(s))^{2} d s$, we can assume $\alpha \equiv 1$. We set functionals $\Gamma_{1}$ and $\Gamma_{2}$ defined on $\left\{w \in \mathscr{W}^{1} ; w(0)=0\right\}$ by

$$
\Gamma_{1}(w)(t) \equiv w(t)-\min _{0 \leqq s \leqq t} w(s), \quad \Gamma_{2}(w)(t) \equiv-\min _{0 \leqq s \leqq t} w(s)
$$

Note that $\left[\Gamma_{1}(w), \Gamma_{2}(w)\right]$ is the unique solution of the Skorohod equation for 0 and $w$ (cf. [5] or Lemma III-4.2 in [2]). Now we define a sequence of $\mathscr{F}_{t^{-}}$ stopping times $\left\{\tau_{n}\right\}_{n=0}^{\infty}$ as follows:

$$
\begin{aligned}
& \tau_{0}=\inf \{t \geqq 0 ; Y(t) \in \mathbb{Z}\} \\
& \tau_{n}= \begin{cases}\inf \left\{t \geqq \tau_{n-1} ;\left|Y(t)-Y\left(\tau_{n-1}\right)\right|=1\right\} & \text { if } \tau_{n-1}<+\infty \\
+\infty & \text { if } \tau_{n-1}=+\infty \quad n \geqq 1 .\end{cases}
\end{aligned}
$$

Then we can observe that with probability one,

$$
\tau_{n}<+\infty \quad n \geqq 0
$$




$$
\begin{aligned}
& \tau_{0}=\inf \{t \geqq 0 ; Y(0)+B(t) \in \mathbb{Z}\} \\
& \begin{array}{l}
\tau_{n+1}=\tau_{n}+\inf \left\{t \geqq 0 ; \Gamma_{1}\left(\stackrel{\circ}{B}_{n}\right)(t)=1\right\} \\
\quad=\tau_{n}+\inf \left\{t \geqq 0 ; \Gamma_{1}\left(B_{n}\right)(t)=1\right\} \quad n \geqq 0
\end{array} \\
& \begin{array}{l}
L_{t \wedge\left(\tau_{n+1}\left(\tau_{n}\right)\right.}\left(Y\left(\cdot+\tau_{n}\right)\right)=\Gamma_{2}\left(\stackrel{\circ}{B}_{n}\right)(t) \quad n \geqq 0
\end{array}
\end{aligned}
$$

where $B_{n}(t) \equiv \int_{0}^{t} \operatorname{sgn}\left(Y\left(s+\tau_{n}\right)-Y\left(\tau_{n}\right)\right) d B\left(s+\tau_{n}\right) \quad$ and $\quad \dot{B}_{n}(t) \equiv B_{n}\left(t \wedge\left(\tau_{n+1}-\tau_{n}\right)\right)$. Indeed, they are shown as follows: From the fact

$$
L_{t \wedge \tau_{0}}^{\delta}(Y)=0 \quad \text { for any } t \geqq 0,
$$

it immediately follows that $\tau_{0}<+\infty$ and (3.3) holds a.s. (P). To see (3.2), (3.4) and (3.5), we assume that (3.2) is true for $n=k(k \geqq 0)$. Since

$$
L_{t}^{l}(Y) \text { is flat on }\left[\tau_{k}, \tau_{k+1}\right) \text { for any } l \in \mathbb{Z} \backslash\left\{Y\left(\tau_{k}\right)\right\},
$$

we see by Itô's formula that

$$
\left|Y\left(t \wedge\left(\tau_{k+1}-\tau_{k}\right)+\tau_{k}\right)-Y\left(\tau_{k}\right)\right|=\stackrel{\circ}{B}_{k}(t)+L_{t \wedge\left(\tau_{k+1}-\tau_{k}\right)}^{Y\left(\tau_{k}\right)}\left(Y\left(\cdot+\tau_{k}\right)\right) .
$$

This expression implies that

$$
\begin{aligned}
& \left|Y\left(t \wedge\left(\tau_{k+1}-\tau_{k}\right)+\tau_{k}\right)-Y\left(\tau_{k}\right)\right|=\Gamma_{1}\left(\stackrel{B}{B}_{k}\right)(t)=\Gamma_{1}\left(B_{k}\right)\left(t \wedge\left(\tau_{k+1}-\tau_{k}\right)\right) \\
& L_{t \wedge\left(\tau_{k+1}-\tau_{k}\right)}^{Y\left(\tau_{k}\right)}\left(Y\left(\cdot+\tau_{k}\right)\right)=\Gamma_{2}\left(\stackrel{B}{B}_{k}\right)(t)=\Gamma_{2}\left(B_{k}\right)\left(t \wedge\left(\tau_{k+1}-\tau_{k}\right)\right) .
\end{aligned}
$$

Hence, noting

$$
B_{k} \text { is an } \mathscr{F}_{t+\tau_{k}}-B M_{0}^{1} \text {, }
$$

we see that (3.2) is true for $n=k+1$, and so are (3.4) and (3.5) for $n=k$. Thus, putting all this together, we conclude that (3.2), (3.4) and (3.5) hold for any $n \geqq 0$.

From (3.6) and (3.7), it is easily seen that

$$
L_{t}^{\delta} Z(Y)=\sum_{k=0}^{\infty} L_{\left(t-\tau_{k}\right)+\wedge\left(\tau_{k+1}-\tau_{k}\right)}^{Y\left(\tau_{k}\right)}\left(Y\left(\cdot+\tau_{k}\right)\right.
$$

By (3.4) and (3.5), this implies that for every $t \geqq 0$

$$
L_{t}^{\delta} \mathbf{z}(Y)=\sum_{k=0}^{\infty} \Gamma_{2}\left(\stackrel{\circ}{B}_{k}\right)\left(\left(t-\tau_{0}-\sum_{j=1}^{k-1} \inf \left\{s \geqq 0 ; \Gamma_{1}\left(\stackrel{\circ}{B}_{j}\right)(s)=1\right\}\right)^{+}\right) .
$$

We here note that $\tau_{0}, \stackrel{\circ}{B}_{0}, \stackrel{\circ}{B}_{1}, \ldots$ are independent and $\dot{B}_{n}(\cdot)$ is equivalent in law to $B(\cdot \wedge \tau)$, where $\tau=\inf \left\{t \geqq 0 ; \Gamma_{1}(B)(t)=1\right\}$. This is derived from (3.4), (3.8) and the $\mathscr{F}_{\tau_{n+1}}$-measurability of $\dot{B}_{n}$. By the same way as above, for $Y(0)+B(\cdot)$ we can observe that for every $t \geqq 0$

$$
L_{t}^{\delta} z(Y(0)+B)=\sum_{k=0}^{\infty} \Gamma_{2}\left(A_{k}\right)\left(\left(t-\tau_{0}-\sum_{j=1}^{k-1} \inf \left\{s \geqq 0 ; \Gamma_{1}\left(A_{j}\right)(s)=1\right\}\right)^{+}\right)
$$


where $\left\{A_{n}\right\}_{n \geqq 0}$ possesses the same property as $\left\{\AA_{n}\right\}_{n \geqq 0}$. Thus we see that the law of $L^{\delta}{ }^{\delta}(Y)$ coincides with one of $L^{\delta} z(Y(0)+B)$, and if we show that for $p>0$ and $t, t^{\prime} \geqq 0$

$$
E\left[\left|L_{t^{\prime}}^{\delta} Z(Y(0)+B)-L_{t}^{\delta} Z(Y(0)+B)\right|^{2 p}\right] \leqq \text { const. }\left(1+\left|t^{\prime}-t\right|^{p}\right)\left|t^{\prime}-t\right|^{p}
$$

where the const. depends only on $p$, then the proof of the proposition is completed.

We prove (3.9). By applying Itô's formula to $Z(t) \equiv Y(0)+B(t)$ with a function $2 \int_{0}^{\eta}(b-[b]) d b$, it is easy to see that for $0 \leqq t \leqq t^{\prime}$

$$
2 \int_{Z(t)}^{Z\left(t^{\prime}\right)}(b-[b]) d b=2 \int_{t}^{t^{\prime}}(Z(s)-[Z(s)]) d B(s)+t^{\prime}-t-\left(L_{t^{\prime}}^{\partial}(Z)-L_{t}^{\delta_{Z}}(Z)\right) .
$$

Hence, we have that for $p>0$

$$
\begin{aligned}
& \left|L_{t^{\prime}}^{\delta}(Z)-L_{t}^{\delta} Z(Z)\right|^{2 p} \\
& \quad \leqq \text { const. }\left\{\left|t^{\prime}-t\right|^{2 p}+\left|B\left(t^{\prime}\right)-B(t)\right|^{2 p}+\left|\int_{t}^{t^{\prime}}(Z(s)-[Z(s)]) d B(s)\right|^{2 p}\right\} 0 \leqq t \leqq t^{\prime}
\end{aligned}
$$

where the const. depends only on $p$, and therefore, taking the expectation, we obtain (3.9) at once.

As a corollary to Proposition 3.1, we have

Corollary 3.1. Let $a, b, \tau, \beta$ and $\mu$ satisfy $(0.2)^{\prime},(0.3),(0.4)$ and $(0.5)$. Then, for any probability measure $\lambda(d x)$ on $\mathbb{R}^{d}$ and any $[X(t), B(t), M(t)] \epsilon$ $\mathbb{S}_{\lambda}[a, b, \tau, \beta, \mu]$, the following estimate holds: For $p>0$

$$
E\left[\left|\sum_{k \in Z} L_{t^{\prime}}^{a_{k}}\left(X^{d}\right)-\sum_{k \in \mathbf{Z}} L_{t}^{a_{k}}\left(X^{d}\right)\right|^{2 p}\right] \leqq \text { const. }\left(1+\left|t^{\prime}-t\right|^{p}\right)\left|t^{\prime}-t\right|^{p} \quad t, t^{\prime} \geqq 0
$$

where $\left\{a_{k}\right\}_{k \in \mathbb{Z}}$ is a sequence appearing in (0.5) and the const. depends only on $p, \inf _{k \in \mathbb{Z}}\left(a_{k+1}-a_{k}\right)$ and $\sup _{k \in \mathbf{Z}}\left(a_{k+1}-a_{k}\right)$.

Proof. Let $[X(t), B(t), M(t)] \in \mathbb{S}_{\lambda}[a, b, \tau, \beta, \mu]$ be defined on a filtered probability space $\left(\Omega, \mathscr{F}, P, \mathscr{F}_{t}\right)$. We define $F(\eta) \in C\left(\mathbb{R}^{1}\right)$ as follows:

$$
F(\eta)=\frac{\eta-a_{k}}{a_{k+1}-a_{k}}(k+1)+\frac{a_{k+1}-\eta}{a_{k+1}-a_{k}} k \quad a_{k} \leqq \eta \leqq a_{k+1} .
$$

Then

$$
\begin{gathered}
F^{+}(\eta)=\frac{1}{a_{k+1}-a_{k}} \quad a_{k} \leqq \eta<a_{k+1}, F^{-}(\eta)=\frac{1}{a_{k+1}-a_{k}} \quad a_{k}<\eta \leqq a_{k+1} \\
F^{\prime \prime}(d \eta)=\sum_{k \in \mathbf{Z}}\left(\frac{1}{a_{k+1}-a_{k}}-\frac{1}{a_{k}-a_{k-1}}\right) \delta_{a_{k}}(d \eta)
\end{gathered}
$$


where $F^{+}\left(F^{-}\right)$denotes the right (left) derivative of $F$ and $F^{\prime \prime}(d \eta)$ denotes the second derivative of $F$ in the distribution sense. Applying Itô's formula to $X^{d}(t)$ with this $F$, we see from (3.11) and (3.12) that

$$
\begin{aligned}
& F\left(X^{d}(t)\right)=F\left(X^{d}(0)\right)+\int_{0}^{t} F^{+}\left(X^{d}(s)\right) d B^{d}(s) \\
& \quad+\frac{1}{2} \sum_{k \in \mathbb{Z}} \int_{0}^{t}\left\{\left(\frac{1}{a_{k+1}-a_{k}}+\frac{1}{a_{k}-a_{k-1}}\right) c_{k} \beta^{d}\left(\Xi(s), a_{k}\right)\right. \\
& \left.\quad+\left(-\frac{1}{a_{k+1}-a_{k}}-\frac{1}{a_{k}-a_{k-1}}\right)\right\} d L_{s}^{a_{k}}\left(X^{d}\right) .
\end{aligned}
$$

Hence, by noting that for each $k \in \mathbb{Z}$

(3.14) $\quad L_{t}^{k}\left(F\left(X^{d}\right)\right)$

$$
\begin{array}{r}
=\frac{1}{2} \int_{0}^{t}\left(\frac{1}{a_{k+1}-a_{k}}-\left(1+c_{k} \beta^{d}\left(\Xi(s), a_{k}\right)\right)+\frac{1}{a_{k}-a_{k-1}}-\left(1-c_{k} \beta^{d}\left(\Xi(s), a_{k}\right)\right)\right) \\
d L_{s}^{a_{k}}\left(X^{d}\right),
\end{array}
$$

(3.13) becomes

$$
F\left(X^{d}(t)\right)=F\left(X^{d}(0)\right)+\int_{0}^{t} F^{+} \circ F^{-1}\left(F\left(X^{d}(s)\right)\right) d B^{d}(s)+\sum_{k \in \mathbb{Z}} \int_{0}^{t} \gamma(s, k) d L_{s}^{k}\left(F\left(X^{d}\right)\right)
$$

where

$$
\gamma(s, k) \equiv \frac{\left(a_{k}-a_{k-1}\right)\left(1+c_{k} \beta^{d}\left(\Xi(s), a_{k}\right)\right)-\left(a_{k+1}-a_{k}\right)\left(1-c_{k} \beta^{d}\left(\Xi(s), a_{k}\right)\right)}{\left(a_{k}-a_{k-1}\right)\left(1+c_{k} \beta^{d}\left(\Xi(s), a_{k}\right)\right)+\left(a_{k+1}-a_{k}\right)\left(1-c_{k} \beta^{d}\left(\Xi(s), a_{k}\right)\right.} .
$$

Thus, we can apply Proposition 3.1 to observe that for $p>0$

$$
E\left[\left|L_{t^{\prime}}^{\delta} \mathbf{z}\left(F\left(X^{d}\right)\right)-L_{t}^{\delta} \mathbf{z}\left(F\left(X^{d}\right)\right)\right|^{2 p}\right] \leqq \text { const. }\left(1+\left|t^{\prime}-t\right|^{p}\right)\left|t^{\prime}-t\right|^{p} \quad t, t^{\prime} \geqq 0
$$

where the const. depends only on $p$ and $\inf _{k \in \mathbf{Z}}\left(a_{k+1}-a_{k}\right)$. Since $d L_{t}^{a_{k}}\left(X^{d}\right)$ $\leqq \sup _{l \in \mathbf{Z}}\left(a_{l+1}-a_{l}\right) d L_{t}^{k}\left(F\left(X^{d}\right)\right)$ for every $k \in \mathbb{Z}$, which is clear from (3.14), the above estimate implies (3.10).

Remark 3.1. For the process $X(t)$ considered in the above, we define a sequence of $\mathscr{F}_{t}$-stopping times $\left\{\tau_{n}\right\}_{n=0}^{\infty}$ by

$$
\begin{aligned}
& \tau_{0}=\inf \left\{t \geqq 0 ; X^{d}(t) \in \mathbb{F}\right\} \\
& \tau_{n}= \begin{cases}\inf \left\{t \geqq \tau_{n-1} ; X^{d}(t) \in \mathbb{F} \backslash\left\{X^{d}\left(\tau_{n-1}\right)\right\}\right\} & \text { if } \tau_{n-1}<+\infty \\
+\infty & \text { if } \tau_{n-1}=+\infty \quad n \geqq 1\end{cases}
\end{aligned}
$$

where $\mathbb{F}=\left\{a_{k} ; k \in \mathbb{Z}\right\}$. Then, with probability one, it holds that $\tau_{n}<+\infty(n \geqq 0)$ and $\lim _{n \uparrow \infty} \tau_{n}=+\infty$, because $\left\{\tau_{n}\right\}_{n=0}^{\infty}$ defined in the proof of Proposition 3.1 has this property. 
Now, using Corollary 3.1 and the result of [6], we prove the nonemptiness of $\mathbb{S}_{\lambda}[a, b, \tau, \beta, \mu]$. Here $a, b, \tau, \beta$ and $\mu$ satisfy $(0.2)^{\prime},(0.3),(0.4)$ and $(0.5)$, and $\lambda(d x)$ is a probability measure on $\mathbb{R}^{d}$.

Proof of Theorem A. Let $\left\{a_{k}\right\}_{k \in \mathbb{Z}}$ and $\left\{c_{k}\right\}_{k \in \mathbb{Z}}$ be sequences appearing in (0.5). For each $\varepsilon>0$ we take a bounded continuous function $\beta_{\varepsilon}: \mathbb{R}^{d} \rightarrow \mathbb{R}^{d}$ and a sequence $\left\{c_{\varepsilon, k}\right\}_{k \in \mathbb{Z}}$ satisfying $c_{\varepsilon, k}>0(k \in \mathbb{Z})$ and $\sum_{k \in \mathbb{Z}} c_{\varepsilon, k}<+\infty$ such that

$$
\begin{aligned}
& \sup _{k \in \mathbb{Z}}\left|c_{\varepsilon, k}-c_{k}\right| \longrightarrow 0, \quad\left\|\beta_{\varepsilon}-\beta\right\|_{\infty} \longrightarrow 0 \quad \text { as } \varepsilon \downarrow 0 \\
& \sup _{\xi \in \mathbb{R}^{d-1}}\left|\beta_{\varepsilon}^{d}\left(\xi, a_{k}\right) c_{\varepsilon, k}\right|<1 \quad \text { for every } k \in \mathbb{Z} .
\end{aligned}
$$

Such a $\beta_{\varepsilon}$ and a $\left\{c_{\varepsilon, k}\right\}_{k \in \mathbb{Z}}$ obviously exist. From (3.15), there exist bounded continuous functions $\bar{\beta}_{\varepsilon}, \bar{\beta}: \mathbb{R}^{d} \rightarrow \mathbb{R}^{d}$ and $\bar{\tau}_{\varepsilon}, \bar{\tau}: \mathbb{R}^{d} \rightarrow \mathbb{R}^{d} \otimes \mathbb{R}^{d}$ satisfying $\bar{\tau}_{\varepsilon j}^{d}=0$ and $\bar{\tau}_{j}^{d}=0(j=1, \ldots, d)$ such that

$$
\begin{aligned}
& \left\|\bar{\beta}_{\varepsilon}-\bar{\beta}\right\|_{\infty} \longrightarrow 0, \quad\left\|\bar{\tau}_{\varepsilon}-\bar{\tau}\right\|_{\infty} \longrightarrow 0 \quad \text { as } \quad \varepsilon \downarrow 0 \\
& \begin{cases}\bar{\beta}_{\varepsilon}\left(\xi, a_{k}\right)=c_{\varepsilon, k} \beta_{\varepsilon}\left(\xi, a_{k}\right), & \bar{\beta}\left(\xi, a_{k}\right)=c_{k} \beta\left(\xi, a_{k}\right) \\
\bar{\tau}_{\varepsilon}\left(\xi, a_{k}\right)=\sqrt{c_{\varepsilon, k}} \tau\left(\xi, a_{k}\right), & \bar{\tau}\left(\xi, a_{k}\right)=\sqrt{c_{k}} \tau\left(\xi, a_{k}\right) \\
& \text { for every } \xi \in \mathbb{R}^{d-1} \text { and } k \in \mathbb{Z} .\end{cases}
\end{aligned}
$$

Now, by virtue of (3.16), on a filtered probability space $\left(\Omega_{\varepsilon}, \mathscr{F}_{\varepsilon}, P_{\varepsilon}, \mathscr{F}_{\varepsilon, t}\right)$, we take a $\left[X_{\imath}(t), B_{\varepsilon}(t), M_{\varepsilon}(t)\right] \in \mathbb{S}_{\lambda}\left[a, b, \tau, \beta_{\varepsilon}, \mu_{\varepsilon}\right]$, where $\mu_{\varepsilon}(d \eta)=\sum_{k \in \mathbb{Z}} c_{\varepsilon, k} \delta_{a_{k}}(d \eta)$ (cf. [6]). Since $a, b, \tau, \beta_{\varepsilon}$ and $\mu_{\varepsilon}$ satisfy $(0.2)^{\prime},(0.3),(0.4)$ and $(0.5)$, by Corollary 3.1 we have the following estimate

$$
E_{\varepsilon}\left[\left|\sum_{k \in \mathbb{Z}} L_{t^{\prime}{ }^{k}}\left(X_{\varepsilon}^{d}\right)-\sum_{k \in \mathbb{Z}} L_{t}^{a_{k}}\left(X_{\varepsilon}^{d}\right)\right|^{8}\right] \leqq \text { const. }\left(1+\left|t^{\prime}-t\right|^{4}\right)\left|t^{\prime}-t\right|^{4} \quad t, t^{\prime} \geqq 0
$$

where the const. depends only on $\inf _{k \in \mathbb{Z}}\left(a_{k+1}-a_{k}\right)$ and $\sup _{k \in \mathbb{Z}}\left(a_{k+1}-a_{k}\right)$. Also, if we define an $\mathscr{F}_{\varepsilon, t}$-martingale $N_{\varepsilon}^{i}(i=1, \ldots, d)$ by

$$
N_{\varepsilon}^{i}(t) \equiv \int_{0}^{t} \sum_{k \in \mathbb{Z}} \chi_{\left\{a_{k}\right\}}\left(X_{\varepsilon}^{d}(s)\right) \frac{1}{\sqrt{c_{\varepsilon, k}}} d M_{\varepsilon}^{i}(s)
$$

and we set $\mathbb{L}_{\varepsilon}(t) \equiv \sum_{k \in \mathbb{Z}} L_{t}^{a_{k}}\left(X_{\varepsilon}^{d}\right)$ for simplicity, then

$$
\begin{aligned}
& \left\langle B_{\varepsilon}^{i}, N_{\varepsilon}^{j}\right\rangle(t)=0, \quad\left\langle N_{\varepsilon}^{i}, N_{\varepsilon}^{j}\right\rangle(t)=\delta_{j}^{i} L_{\varepsilon}(t) \quad i, j=1, \ldots, d, \\
& X_{\varepsilon}^{i}(t)=X_{\varepsilon}^{i}(0)+\sum_{j=1}^{d} \int_{0}^{t} a_{j}^{i}\left(X_{\varepsilon}(s)\right) d B_{\varepsilon}^{j}(s)+\int_{0}^{t} b^{i}\left(X_{\varepsilon}(s)\right) d s \\
& \quad+\sum_{j=1}^{d} \int_{0}^{t} \bar{\tau}_{\varepsilon j}^{i}\left(X_{\varepsilon}(s)\right) d N_{\varepsilon}^{j}(s)+\int_{0}^{t} \bar{\beta}_{\varepsilon}^{i}\left(X_{\varepsilon}(s)\right) d L_{\varepsilon}(s) \quad i=1, \ldots, d
\end{aligned}
$$

where we have derived (3.21) using (3.18). Hence, by (3.19), (3.20) and (3.21), we 
have the tightness of $\left\{\left[X_{\varepsilon}(t), B_{\varepsilon}(t), N_{\varepsilon}(t), L_{\varepsilon}(t)\right]\right\}_{\varepsilon}>0$.

In the following we trace the same argument as done in [6]. We choose a positive sequence $\left\{\varepsilon_{n}\right\}_{n=1}^{\infty}$ tending to zero such that the continuous process $\left[X_{\varepsilon_{n}}(t)\right.$, $\left.B_{\varepsilon_{n}}(t), N_{\varepsilon_{n}}(t), L_{\varepsilon_{n}}(t)\right]$ converges in law to a continuous process $[X(t), B(t)$, $N(t), L(t)]$ as $n \uparrow \infty$. By the celebrated theorem due to Skorohod, we may assume the following:

(i) $\left(\Omega_{\varepsilon_{n}}, \mathscr{F}_{\varepsilon_{n}}, P_{\varepsilon_{n}}\right)=(\Omega, \mathscr{F}, P)$ for $n \geqq 1$, and $[X(t), B(t), N(t), L(t)]$ is defined on this probability space $(\Omega, \mathscr{F}, P)$.

(ii) $\left[X_{\varepsilon_{n}}(t), B_{\varepsilon_{n}}(t), N_{\varepsilon_{n}}(t), L_{\varepsilon_{n}}(t)\right]$ converges to $[X(t), B(t), N(t), L(t)]$ uniformly in $t$ on every finite interval as $n \uparrow \infty$. We set $\mathscr{F}_{t} \equiv \cap_{\delta>0} \sigma(X(s), B(s)$, $N(s), L(s) ; 0 \leqq s \leqq t+\delta)$. Clearly $L(t)$ is an $\mathscr{F}_{t}$-adapted continuous increasing process with $L(0)=0$. Since $\left\langle B_{\varepsilon_{n}}^{i}, B_{\varepsilon_{n}}^{j}\right\rangle(t)=\delta_{j}^{i} t(i, j=1, \ldots, d)$ and (3.20) holds for $\varepsilon=\varepsilon_{n}$, we see from (3.19) and the above (ii) that $[B(t), N(t)]$ is a system of $\mathscr{F}_{t^{-}}$ martingales with $B(0)=N(0)=0$ such that $\left\langle B^{i}, B^{j}\right\rangle(t)=\delta_{j}^{i} t,\left\langle B^{i}, N^{j}\right\rangle(t)=0$ and $\left\langle N^{i}, N^{j}\right\rangle(t)=\delta_{j}^{i} L(t)$ for $i, j=1, \ldots, d$. Hence, applying Proposition 2 in [6], we observe from (ii) and (3.17) that as $n \uparrow \infty$, (3.21) becomes

$$
\begin{aligned}
X^{i}(t)= & X^{i}(0)+\sum_{j=1}^{d} \int_{0}^{t} a_{j}^{i}(X(s)) d B^{j}(s)+\int_{0}^{t} b^{i}(X(s)) d s \\
& +\sum_{j=1}^{d} \int_{0}^{t} \bar{\tau}_{j}^{i}(X(s)) d N^{j}(s)+\int_{0}^{t} \bar{\beta}^{i}(X(s)) d L(s) \quad i=1, \ldots, d,
\end{aligned}
$$

which especially implies that $X(t)$ is a system of $\mathscr{F}_{t}$-semimartingales. It remains to show that

$$
L(t)=\sum_{k \in \mathbf{Z}} L_{t}^{a_{k}}\left(X^{d}\right) \quad t \geqq 0 .
$$

If this is true, then, by setting $M(t) \equiv \int_{0}^{t} \sum_{k \in \mathbf{Z}} \chi_{\left\{a_{k}\right\}}\left(X^{d}(s)\right) \sqrt{c_{k}} d N(s),[X(t), B(t)$, $M(t)] \in \boldsymbol{S}_{\lambda}[a, b, \tau, \beta, \mu]$ follows from (3.18).

To prove (3.22), it is sufficient to show that for each $k \in \mathbb{Z}$

$$
\underset{n \uparrow \infty}{\text { 1.i.p. }} \max _{0 \leqq t \leqq T}\left|L_{t}^{a_{k}}\left(X_{\varepsilon_{n}}^{d}\right)-L_{t}^{a_{k}}\left(X^{d}\right)\right|=0 \quad T>0,
$$

because (3.22) is the implication of this, (3.30) and (3.28)' below. Now, applying Itô's formula to (3.21) for $i=d$ and (3.21)' for $i=d$, respectively, we see that for each $\eta \in \mathbb{R}^{1}$

$$
L_{t}^{\eta}\left(X_{\varepsilon_{n}}^{d}\right)=\left|X_{\varepsilon_{n}}^{d}(t)-\eta\right|-\left|X_{\varepsilon_{n}}^{d}(0)-\eta\right|-\int_{0}^{t} \operatorname{sgn}\left(X_{\varepsilon_{n}}^{d}(s)-\eta\right) d B_{\varepsilon_{n}}^{d}(s)
$$




$$
\begin{aligned}
& -\int_{0}^{t} \operatorname{sgn}\left(X_{\varepsilon_{n}}^{d}(s)-\eta\right) \bar{\beta}_{\varepsilon_{n}}^{d}\left(X_{\varepsilon_{n}}(s)\right) d L_{\varepsilon_{n}}(s) \\
= & :\left|X_{\varepsilon_{n}}^{d}(t)-\eta\right|-\left|X_{\varepsilon_{n}}^{d}(0)-\eta\right|-I_{n}(\eta ; t)-V_{n}(\eta ; t) \\
L_{t}^{\eta}\left(X^{d}\right)= & \left|X^{d}(t)-\eta\right|-\left|X^{d}(0)-\eta\right|-\int_{0}^{t} \operatorname{sgn}\left(X^{d}(s)-\eta\right) d B^{d}(s) \\
& -\int_{0}^{t} \operatorname{sgn}\left(X^{d}(s)-\eta\right) \bar{\beta}^{d}(X(s)) d L(s) \\
= & :\left|X^{d}(t)-\eta\right|-\left|X^{d}(0)-\eta\right|-I(\eta ; t)-V(\eta ; t) .
\end{aligned}
$$

By (3.19) and the same estimate for $L(\cdot)$, which is clear from (3.19), (3.24) and (3.24)' yield the following estimates:

$$
\sup _{\eta \in \mathbb{R}^{1}} E\left[L_{t}^{\eta}\left(X_{\varepsilon_{n}}^{d}\right)\right], \quad \sup _{\eta \in \mathbb{R}^{1}} E\left[L_{t}^{\eta}\left(X^{d}\right)\right] \leqq \text { const. }(1+\sqrt{t}) \sqrt{t} \quad t \geqq 0
$$

where the const. depends only on $\inf _{k \in \mathbf{Z}}\left(a_{k+1}-a_{k}\right), \sup _{k \in \mathbb{Z}}\left(a_{k+1}-a_{k}\right)$ and $\sup _{n \geqq 1}\left\|\bar{\beta}_{\varepsilon_{n}}^{d}\right\|_{\infty}$. By these estimates and Proposition 2 in [6], we can observe that for each $\eta \in \mathbb{R}^{1}$ and $T>0$

$$
\lim _{n \uparrow \infty} E\left[\max _{0 \leqq t \leqq T}\left|I_{n}(\eta ; t)-I(\eta ; t)\right|^{2}\right]=0 .
$$

In fact, if we choose $p_{\delta} \in C_{b}\left(\mathbb{R}^{1}\right)$ for $\delta>0$ such that $\left|p_{\delta}(\eta)-\operatorname{sgn}(\eta)\right| \leqq \chi_{(-\delta, \delta)}(\eta)$ for $\eta \in \mathbb{R}^{1}$, then by (3.25)

$$
\begin{aligned}
& \lim _{\delta \downarrow 0} \limsup _{n \uparrow \infty} E\left[\max _{0 \leqq t \leqq T}\left|I_{n}(\eta ; t)-\int_{0}^{t} p_{\delta}\left(X_{\varepsilon_{n}}^{d}(s)-\eta\right) d B_{\varepsilon_{n}}^{d}(s)\right|^{2}\right]=0 \\
& \lim _{\delta \downarrow 0} E\left[\max _{\llcorner 0 \leqq t \leqq T}\left|I(\eta ; t)-\int_{0}^{t} p_{\delta}\left(X^{d}(s)-\eta\right) d B^{d}(s)\right|^{2}\right]=0
\end{aligned}
$$

and by Proposition 2 in [6]

$$
\lim _{n \uparrow 0} E\left[\max _{0 \leqq t \leqq T}\left|\int_{0}^{t} p_{\delta}\left(X_{\varepsilon_{n}}^{d}(s)-\eta\right) d B_{\varepsilon_{n}}^{d}(s)-\int_{0}^{t} p_{\delta}\left(X^{d}(s)-\eta\right) d B^{d}(s)\right|^{2}\right]=0 .
$$

Hence, by putting all this together, (3.26) follows immediately. On the other hand, we can also observe that for each $k \in \mathbb{Z}$

$$
V_{n}\left(a_{k} ; \cdot\right) \underset{c}{\longrightarrow} V\left(a_{k} ; \cdot\right) \quad \text { as } n \uparrow \infty .
$$

Here " $\underset{c}{\rightrightarrows}$ " denotes the compact uniform convergence in $t$. Indeed, (3.27) is shown in the following way: Since

$$
\begin{aligned}
& L_{\varepsilon_{n}}(t)=\sum_{k \in \mathbb{Z}} L_{t}^{a_{k}}\left(X_{\varepsilon_{n}}^{d}\right) \quad t \geqq 0 \\
& L_{\varepsilon_{n}}(\cdot) \underset{c}{\longrightarrow} L(\cdot) \quad \text { as } n \uparrow \infty,
\end{aligned}
$$


for any subsequence $\left\{n^{\prime}\right\}$ of $\{n\}$, we find a further subsequence $\left\{n^{\prime \prime}\right\}$ of $\left\{n^{\prime}\right\}$ and a continuous increasing function $L^{k}(t)(k \in \mathbb{Z})$ such that

$$
L^{a_{k}}\left(X_{\varepsilon_{n^{\prime \prime}}}^{d}\right) \underset{c}{\longrightarrow} L^{k}(\cdot) \quad \text { as } \quad n^{\prime \prime} \uparrow \infty .
$$

We note that $L^{k}(\cdot)$ has the following property:

$$
\int_{0}^{t} \chi_{\left\{a_{k}\right\}}\left(X^{d}(s)\right) d L^{k}(s)=L^{k}(t) \quad t \geqq 0
$$

Hence, letting $n^{\prime \prime} \uparrow \infty$ in (3.28), we see from (3.29), (3.30) and (3.31) that

$$
L(t)=\sum_{k \in \mathbf{Z}} L^{k}(t) \quad t \geqq 0 .
$$

Therefore, since

$$
V_{n}\left(a_{k} ; t\right)=\sum_{l>k} \int_{0}^{t} \bar{\beta}_{\varepsilon_{n}}^{d}\left(X_{\varepsilon_{n}}(s)\right) d L_{s}^{a_{l}}\left(X_{\varepsilon_{n}}^{d}\right)-\sum_{l<k} \int_{0}^{t} \bar{\beta}_{\varepsilon_{n}}^{d}\left(X_{\varepsilon_{n}}(s)\right) d L_{s}^{a_{l}}\left(X_{\varepsilon_{n}}^{d}\right),
$$

by (3.17), (3.30) and $(3.28)^{\prime}, V_{n^{\prime \prime}}\left(a_{k} ; \cdot\right) \underset{c}{\rightrightarrows} V\left(a_{k} ; \cdot\right)$ as $n^{\prime \prime} \uparrow \infty$, which implies (3.27) from the arbitrariness of $\left\{n^{\prime}\right\}$. Consequently, combining (3.26) and (3.27), we immediately have (3.23) from (3.24) and (3.24)'. Thus we conclude the proof of Theorem A.

\section{§4. Uniqueness III}

In this section we prove Theorem $B$ in the general case. By the same reason as stated in the beginning of $\S 3$, we have only to show the uniqueness for $\mathbb{S}_{\lambda}[a, b$, $\tau, \beta, \mu]$ in the case when $a, b, \tau, \beta$ and $\mu$ satisfy $(0.2)^{\prime},(0.3),(0.4),(0.5)$ and (0.6), and $\lambda(d x)$ is a probability measure on $\mathbb{R}^{d}$.

Let $a, b, \tau, \beta, \mu$ and $\lambda$ be such those and fixed. Let $\left\{c_{k}\right\}_{k \in \mathbf{Z}}$ and $\left\{a_{k}\right\}_{k \in \mathbf{Z}}$ be sequences appearing in (0.5). For $k \in \mathbb{Z}$ and $x \in \mathbb{R}^{d}, \mathbb{S}_{\delta_{x}}\left[a, b, \tau, \beta, c_{k} \delta_{a_{k}}\right]$ is nonempty and its uniqueness holds from the conclusions in $\S 2$ and $\S 3$. Hence, we denote by $P_{x}^{k}$ the unique probability law of $X(\cdot)$, where $[X(t), B(t), M(t)] \epsilon$ $\boldsymbol{S}_{\delta_{x}}\left[a, b, \tau, \beta, c_{k} \delta_{a_{k}}\right]$. Note that for each $k \in \mathbb{Z}$, a mapping $x \mapsto P_{x}^{k}$ is continuous, which is a consequence of the estimate given in Corollary 3.1 and the uniqueness for $\boldsymbol{S}_{\delta_{x}}\left[a, b, \tau, \beta, c_{k} \delta_{a_{k}}\right]$.

Now, let $[X(t), B(t), M(t)]$ be an element of $\mathbb{S}_{\lambda}[a, b, \tau, \beta, \mu]$ defined on a filtered probability space $\left(\Omega, \mathscr{F}, P, \mathscr{F}_{t}\right)$. Let $\left\{\tau_{n}\right\}_{n=0}^{\infty}$ be a sequence of $\mathscr{F}_{t^{-}}$ stopping times defined in Remark 3.1. Then $\tau_{n}<+\infty(n \geqq 0)$ a.s. $(P)$ and

$$
\lim _{n \uparrow \infty} \tau_{n}=+\infty \quad \text { a.s. }(P) \text {. }
$$


First it is easy to see that $\tau_{0}=\inf \left\{t \geqq 0 ; X^{d}(0)+B^{d}(t) \in \mathbb{F}\right\}$ and

$$
\begin{array}{r}
X^{i}\left(t \wedge \tau_{0}\right)=X^{i}(0)+\sum_{j=1}^{d} \int_{0}^{t} a_{j}^{i}\left(X\left(s \wedge \tau_{0}\right)\right) d B^{j}\left(s \wedge \tau_{0}\right)+\int_{0}^{t} b^{i}\left(X\left(s \wedge \tau_{0}\right)\right) d s \wedge \tau_{0} \\
i=1, \ldots, d
\end{array}
$$

where $\mathbb{F}=\left\{a_{k} ; k \in \mathbb{Z}\right\}$, so that it holds that

$$
P\left(X\left(\cdot \wedge \tau_{0}\right) \in *\right)=\int_{\mathbb{R}^{d}} \stackrel{\circ}{P} x(*) \lambda(d x)
$$

where $\stackrel{\circ}{P}^{x}$ stands for a probability measure on $\mathscr{W}^{d}$ given in $\S \S 1.1$ for $a$ and $b$. Next, in view of $(0.1)^{\prime}$ we observe the following: For each $n \geqq 0$

$$
\begin{gathered}
X^{i}\left(t+\tau_{n}\right)=X^{i}\left(\tau_{n}\right)+\sum_{j=1}^{d} \int_{0}^{t} a_{j}^{i}\left(X\left(s+\tau_{n}\right)\right) d B^{j}\left(s+\tau_{n}\right)+\int_{0}^{t} b^{i}\left(X\left(s+\tau_{n}\right)\right) d s \\
+\sum_{j=1}^{d} \int_{0}^{t} \tau_{j}^{i}\left(X\left(s+\tau_{n}\right)\right) d M^{j}\left(s+\tau_{n}\right)+\int_{0}^{t} \beta^{i}\left(X\left(s+\tau_{n}\right)\right) c_{k_{n}} d L_{s}^{a_{k_{n}}}\left(X^{d}\left(\cdot+\tau_{n}\right)\right) \\
i=1, \ldots, d \quad 0 \leqq t \leqq \tau_{n+1}-\tau_{n}, \\
\left\langle M^{i}\left(\cdot+\tau_{n}\right)-M^{i}\left(\tau_{n}\right), M^{j}\left(\cdot+\tau_{n}\right)-M^{j}\left(\tau_{n}\right)\right\rangle(t)=\delta_{j}^{i} c_{k_{n}} L_{t}^{a_{k_{n}}}\left(X^{d}\left(\cdot+\tau_{n}\right)\right) \\
i, j=1, \ldots, d \quad 0 \leqq t \leqq \tau_{n+1}-\tau_{n}, \\
\tau_{n+1}-\tau_{n}=\inf \left\{s \geqq 0 ; X^{d}\left(s+\tau_{n}\right) \in F \backslash\left\{a_{k_{n}}\right\}\right\} .
\end{gathered}
$$

Here $k_{n}$ is an $\mathscr{F}_{\tau_{n}}$-measurable random variable defined by $a_{k_{n}}=X^{d}\left(\tau_{n}\right)$. Hence, from the uniqueness of $\mathbb{S}_{\delta_{x}}\left[a, b, \tau, \beta, c_{k} \delta_{a_{k}}\right]$ it follows that for each $n \geqq 0$

$$
P\left(X\left(\cdot \wedge\left(\tau_{n+1}-\tau_{n}\right)+\tau_{n}\right) \in * \mid \mathscr{F}_{\tau_{n}}\right)=\left.P_{X\left(\tau_{n}\right)}^{k_{n}}\left(w\left(\cdot \wedge \sigma_{k}\right) \in *\right)\right|_{k=k_{n}} \quad \text { a.s. }(P)
$$

where $\sigma_{k}(w)=\inf \left\{t \geqq 0 ; w^{d}(t) \in \mathbb{F} \backslash\left\{a_{k}\right\}\right\}$ for $w \in \mathscr{W}^{d}$ and $k \in \mathbb{Z}$. Therefore, putting (4.2) and (4.3) together, we can see that for each $n \geqq 0$

the probability law of $X\left(\cdot \wedge \tau_{n}\right)$ is uniquely determined.

Indeed, this is shown by induction on $n(\geqq 0)$ :

(i) For $n=0,(4.4)$ is clear from (4.2).

(ii) We assume that (4.4) is true for $n \leqq p(p \geqq 0)$. Then, since

$$
\begin{aligned}
P\left(X\left(\cdot \wedge \tau_{p+1}\right) \in *\right) & =E\left[P\left(X\left(\left(\cdot-\tau_{p}\right) \wedge\left(\tau_{p+1}-\tau_{p}\right)+\tau_{p}\right) \in * \mid \mathscr{F}_{\tau_{p}}\right)\right] \\
& =E\left[\left.P_{X(\sigma)}^{k}\left(w\left((\cdot-\sigma) \wedge \sigma_{k}\right) \in *\right)\right|_{\sigma=\tau_{p}, k=k_{p}}\right]
\end{aligned}
$$

by using (4.3), our assumption implies that (4.4) is true for $n=p+1$. Hence, from (i) and (ii) it follows that (4.4) holds for any $n \geqq 0$. Consequently, combining (4.1) and (4.4), we conclude that the probability law of $X(\cdot)$ is uniquely determined, and thus the proof of the uniqueness of $\mathbb{S}_{\lambda}[a, b, \tau, \beta, \mu]$ is completed. 


\section{Acknowledgement}

The author wishes to express his hearty thanks to Professor S. Watanabe for his valuable suggestions and to Professor S. Kotani for his encouragement.

\section{References}

[1] Anulova, S. V., Diffusion processes with singular characteristic, Stochastic Differential Systems, Proc. IFIP-WG Conf. Vilnius, 264-269, Lect. Notes in Control and Inf. Sci. 25, Springer-Verlag, Berlin, 1978.

[2] Ikeda, N. and Watanabe, S., Stochastic Differential Equations and Diffusion Processes, Kodansha, Tokyo, 1981.

[3] Le Gall, J. F., Temps locaux et équations différentielles stochastiques, Doctorat thèse de 3e cycle, Université Pierre et Marie Curie, 1982.

[4] Ôshima, Y., Some singular diffusion processes and their associated stochastic differential equations, $Z$. Wahr. verw. Geb., 59 (1982), 249-276.

[5] Skorohod, A. V., Stochastic equations for diffusion processes in a bounded region, Theor. Prob. Appl., 6 (1961), 264-274.

[6] Takanobu, S., On the existence of solutions of stochastic differential equations with singular drifts, to appear in Probab. Th. Rel. Fields.

[7] Watanabe, S., On stochastic differential equations for multidimensional diffusion processes with boundary conditions, J. Math. Kyoto Univ., $\mathbb{1} 1$ (1971), 169-180.

[ 8 ] — Excursion point processes of diffusion and stochastic integral, Proc. Intern. Symp. SDE Kyoto 1976 (ed. by K. Itô), 437-461, Kinokuniya, Tokyo, 1978.

[9] — Construction of diffusion processes with Wentzell's boundary conditions by means of Poisson point processes of Brownian excursions, Probability theory, Banach Center Publications, 5, 255-271, Polish Scientific Publishers, Warsaw, 1979. 